\title{
THE EINSTEIN@HOME GAMMA-RAY PULSAR SURVEY. I. SEARCH METHODS, SENSITIVITY, and DISCOVERY of NEW YOUNG GAMMA-RAY PULSARS
}

DOI:

10.3847/1538-4357/834/2/106

\section{Document Version}

Final published version

Link to publication record in Manchester Research Explorer

Citation for published version (APA):

Clark, C. J., Wu, J., Pletsch, H. J., Guillemot, L., Allen, B., Aulbert, C., Beer, C., Bock, O., Cuéllar, A., Eggenstein, H. B., Fehrmann, H., Kramer, M., Machenschalk, B., \& Nieder, L. (2017). THE EINSTEIN@HOME GAMMA-RAY PULSAR SURVEY. I. SEARCH METHODS, SENSITIVITY, and DISCOVERY of NEW YOUNG GAMMA-RAY PULSARS. Astrophysical Journal, 834(2), [106]. https://doi.org/10.3847/1538-4357/834/2/106

\section{Published in:}

Astrophysical Journal

\section{Citing this paper}

Please note that where the full-text provided on Manchester Research Explorer is the Author Accepted Manuscript or Proof version this may differ from the final Published version. If citing, it is advised that you check and use the publisher's definitive version.

\section{General rights}

Copyright and moral rights for the publications made accessible in the Research Explorer are retained by the authors and/or other copyright owners and it is a condition of accessing publications that users recognise and abide by the legal requirements associated with these rights.

\section{Takedown policy}

If you believe that this document breaches copyright please refer to the University of Manchester's Takedown Procedures [http://man.ac.uk/04Y6Bo] or contact uml.scholarlycommunications@manchester.ac.uk providing relevant details, so we can investigate your claim.

\section{OPEN ACCESS}




\title{
THE EINSTEIN@HOME GAMMA-RAY PULSAR SURVEY. I. SEARCH METHODS, SENSITIVITY, AND DISCOVERY OF NEW YOUNG GAMMA-RAY PULSARS
}

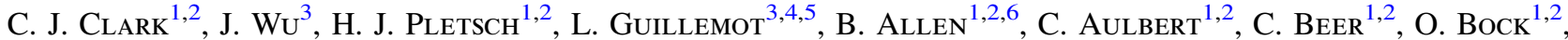 \\ A. Cuéllar ${ }^{1,2}$, H. B. EgGenstein ${ }^{1,2}$, H. FehrmanN ${ }^{1,2}$, M. Kramer $^{3,7,8}$, B. Machenschalk ${ }^{1,2}$, and L. Nieder ${ }^{1,2}$ \\ ${ }^{1}$ Albert-Einstein-Institut, Max-Planck-Institut für Gravitationsphysik, D-30167 Hannover, Germany; colin.clark@aei.mpg.de \\ ${ }^{2}$ Leibniz Universität Hannover, D-30167 Hannover, Germany \\ ${ }^{3}$ Max-Planck-Institut für Radioastronomie, Auf dem Hügel 69, D-53121 Bonn, Germany \\ ${ }^{4}$ Laboratoire de Physique et Chimie de l'Environnement et de l'Espace-Université d'Orléans/CNRS, F-45071 Orléans Cedex 02, France \\ ${ }^{5}$ Station de radioastronomie de Nançay, Observatoire de Paris, CNRS/INSU, F-18330 Nançay, France \\ ${ }^{6}$ Department of Physics, University of Wisconsin-Milwaukee, P.O. Box 413, Milwaukee, WI 53201, USA \\ 7 Jodrell Bank Centre for Astrophysics, School of Physics and Astronomy, The University of Manchester, Manchester M13 9PL, UK \\ ${ }^{8}$ University of Manchester, Manchester M13 9PL, UK \\ Received 2016 May 18; revised 2016 October 5; accepted 2016 October 17; published 2017 January 5
}

\begin{abstract}
We report on the results of a recent blind search survey for gamma-ray pulsars in Fermi Large Area Telescope (LAT) data being carried out on the distributed volunteer computing system, Einstein@Home. The survey has searched for pulsations in 118 unidentified pulsar-like sources, requiring about 10,000 years of CPU core time. In total, this survey has resulted in the discovery of 17 new gamma-ray pulsars, of which 13 are newly reported in this work, and an accompanying paper. These pulsars are all young, isolated pulsars with characteristic ages between $12 \mathrm{kyr}$ and $2 \mathrm{Myr}$, and spin-down powers between $10^{34}$ and $4 \times 10^{36} \mathrm{erg} \mathrm{s}^{-1}$. Two of these are the slowest spinning gamma-ray pulsars yet known. One pulsar experienced a very large glitch $\Delta f / f \approx 3.5 \times 10^{-6}$ during the Fermi mission. In this, the first of two associated papers, we describe the search scheme used in this survey, and estimate the sensitivity of our search to pulsations in unidentified Fermi-LAT sources. One such estimate results in an upper limit of $57 \%$ for the fraction of pulsed emission from the gamma-ray source associated with the Cas A supernova remnant, constraining the pulsed gamma-ray photon flux that can be produced by the neutron star at its center. We also present the results of precise timing analyses for each of the newly detected pulsars.
\end{abstract}

Key words: gamma-rays: stars - pulsars: individual (PSR J0359+5414, PSR J1057-5851, PSR J1350-6225, PSR J1827-1446, PSR J1844-0346)

\section{INTRODUCTION}

Since the launch of the Fermi Gamma-ray Space Telescope in 2008, the on-board Large Area Telescope (LAT; Atwood et al. 2009) has increased the number of known gamma-ray pulsars from around 10 to over $200 .^{9}$ Indeed, thanks to the LAT, we now know pulsars to be the dominant individual gamma-ray source class within the Milky Way galaxy (Second Fermi Large Area Telescope Catalog of Gamma-ray Pulsars, hereafter 2PC, Abdo et al. 2013).

Two-thirds of gamma-ray pulsars were first detected by observations in other wavelength regimes (e.g., radio or X-ray pulsars), the rotation ephemerides from which could then be used to "phase fold" the LAT photon arrival times to test for pulsed gamma-ray emission. However, approximately onethird of the LAT-detected pulsars were unknown prior to the discovery of pulsations in their gamma-ray flux (Abdo et al. 2008, 2009; Saz Parkinson et al. 2010; Pletsch et al. 2012a, 2012b, 2012, 2013). Only a handful of these pulsars were subsequently detected in radio observations, the others could not have been discovered without "blind" searches in gamma-ray data.

The recent Fermi-LAT Third Source Catalog (3FGL; Acero et al. 2015) includes 3033 gamma-ray sources, of which about 1000 lack strong associations or likely counterparts from observations at other wavelengths. Many of these sources have similar properties to the known gamma-ray pulsars (i.e., low time variability and a highly curved spectrum). A large effort is

\footnotetext{
9 http://tinyurl.com/fermipulsars
}

underway to identify pulsars among the unidentified pulsar-like gamma-ray sources, both by performing dedicated radio searches targeting the locations of LAT sources (e.g., Camilo et al. 2015; Cromartie et al. 2016), and by searching among the LAT data itself for pulsations. This paper, and an accompanying paper (J. Wu et al. 2016, in preparation, hereafter Paper II), will describe the latest results from the latter technique.

Due to the sparsity of the LAT photon data (only about 10 photons per day are detected from a typical gamma-ray pulsar), blindly searching for pulsations among the LAT photon arrival times is an enormously expensive computational task. For weak pulsar signals, long integration times are required to reach a detectable signal-to-noise ratio $(\mathrm{S} / \mathrm{N})$, and, as a result, signal parameters must be searched with an extremely fine resolution to avoid losing the signal.

In addition, ensuring sensitivity to the more extreme gammaray pulsars, such as young pulsars with high spin-down rates or faster spinning millisecond pulsars (MSPs), requires searching over vast parameter volumes, and therefore incurs a proportionally large computation cost. To meet these requirements, we utilize the computing power of the Einstein@Home project, which distributes the computations among the many thousands of participating volunteers' devices (Allen et al. 2013). We began performing blind searches for gamma-ray pulsars on Einstein@Home in 2011, with the first such survey resulting in the detection of four new pulsars (Pletsch et al. 2013).

In a recent study (Pletsch \& Clark 2014), we investigated the efficiency of different blind search schemes (e.g., Atwood et al. 2006), and developed new techniques to boost the 
sensitivity of a search without impacting its computational cost. These new methods are currently being used as part of a large-scale survey of pulsar-like Fermi-LAT sources, running on Einstein@Home. In combination with the recent "Pass 8" improvements to the LAT event reconstruction (Atwood et al. 2013), these advances have had a spectacular effect on the blind search sensitivity, an early indication of which was given by the detection of PSR J1906+0722 within one of the first sources searched in this survey (Clark et al. 2015).

In this paper, we present 13 new pulsar discoveries from the fullEinstein@Home survey of 118 sources. These are the result of around 10,000 years of CPU time generously donated by volunteers.

The paper is organized as follows. In Section 2, the search methods are described; an investigation of the sensitivity of the search follows in Section 3; details of the newly discovered pulsars and their timing solutions are given in Section 4; Section 5 contains a discussion of the sensitivity of blind searches to unidentified gamma-ray pulsars; and finally we summarize our conclusions in Section 6.

In Paper II, we will describe in more detail the LAT data preparation procedures; the selection of target sources and ranking based on their spectral properties; the identification of candidate multiwavelength counterparts; phase-resolved gamma-ray spectral analyses of the newly discovered pulsars; and the results of dedicated follow-up radio pulsation searches.

\section{SEARCH SCHEME}

\subsection{Data}

The data searched during the survey consisted of gamma-ray photons detected by the LAT between 2008 August 4 and 2014 April 6 (2014 October 1 for some sources searched later in the survey) with energies above $100 \mathrm{MeV}$. Photons were included if they arrived within $8^{\circ}$ of a target source, with a zenith angle $<100^{\circ}$ and when the LAT's rocking angle was $<52^{\circ}$. The photons were selected and analyzed using the P8_SOURCE_V3 instrument response functions (IRFs).

For each target source, we performed a likelihood spectral analysis using the pointlike package (Kerr 2010). Our source model included all 3FGL catalog sources within $13^{\circ}$ of the target source and used the template_4years_P8_ V2_scaled.fits map cube and isotropic_source_ 4years_P8V3 template to model the Galactic diffuse emission (Acero et al. 2016) and isotropic background respectively.

Target sources were modeled with an exponentially cutoff power law typical of gamma-ray pulsars. During the likelihood fitting, we allowed the normalization of the diffuse models, and the spectral parameters of the target source and all 3FGL sources within $5^{\circ}$ to vary. Sources searched near the beginning of the survey had their sky positions fixed at the 3FGL location. Later sources were relocalized during the likelihood fitting to exploit the improved angular resolution offered by the Pass 8 data. Spectral energy distribution plots and Test Statistic (TS) maps were visually compared to the corresponding 3FGL sources to diagnose any problems with the fitting. With the best-fitting source model, we used gtsrcprob ${ }^{10}$ to compute weights representing the probability of each photon having come from our target source based on their reconstructed

\footnotetext{
${ }^{10}$ gtsrcprob is part of the Fermi Science Tools, available at http://fermi. gsfc.nasa.gov/ssc/data/analysis/software/.
}

energy and arrival direction. Full details of the data preparation methods, and a description of how target sources were prioritized for searching, will be given in Paper II.

The IRFs and diffuse templates used here were internal prerelease versions of the Pass 8 analysis tools because the final release versions were not yet available when the survey began. When investigating the gamma-ray emission from the region surrounding PSR J1906+0722 (Clark et al. 2015), we found that these preliminary IRFs and templates resulted in spectral parameters consistent with those found using the final Pass 8 release. However, photon weights calculated with the most recent Pass 8 data usually result in slightly higher pulsation significance within the same time interval; the sensitivity estimates in Section 3 are likely to be more conservative as a result.

\subsection{Parameter Space}

To search for gamma-ray pulsations in LAT data, it is necessary to assume a certain "phase model" (i.e., a rotation ephemeris) relating the arrival time of every photon to a certain rotational phase, and test all possible combinations of the model parameters for pulsations, indicated by large values of a detection statistic (described in Section 2.3). In the case where a signal is present, the distribution of rotational phases will deviate significantly from uniformity. For isolated pulsars, the phase model $^{11}$ is typically described by a Taylor series expansion in time around a chosen reference epoch $t_{\mathrm{ref}}$, for photon arrival time $t$ at the solar system barycenter (SSB),

$$
\Phi(t)=\Phi_{0}+2 \pi \sum_{m=1} \frac{f^{(m-1)}}{m !}\left(t-t_{\mathrm{ref}}\right)^{m},
$$

where $f^{(m)}$ denotes the $m$ th time derivative of the pulsar's rotational frequency, $f$. While the higher derivative terms are often measurable for young pulsars, it is usually sufficient (and often only feasible) to include only the first two terms in the blind search, resulting in a simplified phase model in which the spin frequency decreases by a constant spin-down rate, $\dot{f} \equiv f^{(1)}$.

Aside from correcting for this constant spin-down, it is also necessary to account for the apparent Doppler modulation of pulsations that results from the Earth's orbit around the SSB. This can be achieved by applying position-dependent corrections to the measured photon arrival times, to retrieve the set of arrival times at the SSB, hereafter denoted as $\left\{t_{j}\right\}$. The angular resolution at which sky positions must be searched increases linearly with the pulsar's spin frequency (see Equation (11)). For all but the slowest of pulsars, the required resolution is finer than the gamma-ray source localization, determined by the LAT's point-spread function. For a blind survey of unidentified gamma-ray point sources, it is therefore necessary to search in two sky positional parameters (R.A. $\alpha$ and decl. $\delta$ ), making the overall search parameter space four-dimensional. For sources for which we used the original 3FGL locations, we searched a circular region around the source with an angular radius that was $50 \%$ larger than the semimajor axis of the $95 \%$ confidence region. For relocalized sources, we searched a conservatively large region with a radius three times larger than the semimajor axis of the $68 \%$ confidence region.

\footnotetext{
${ }^{11}$ While we define the phase in radians, in all plots we show the phase in rotations for clarity, and re-normalize the pulse profiles accordingly.
} 


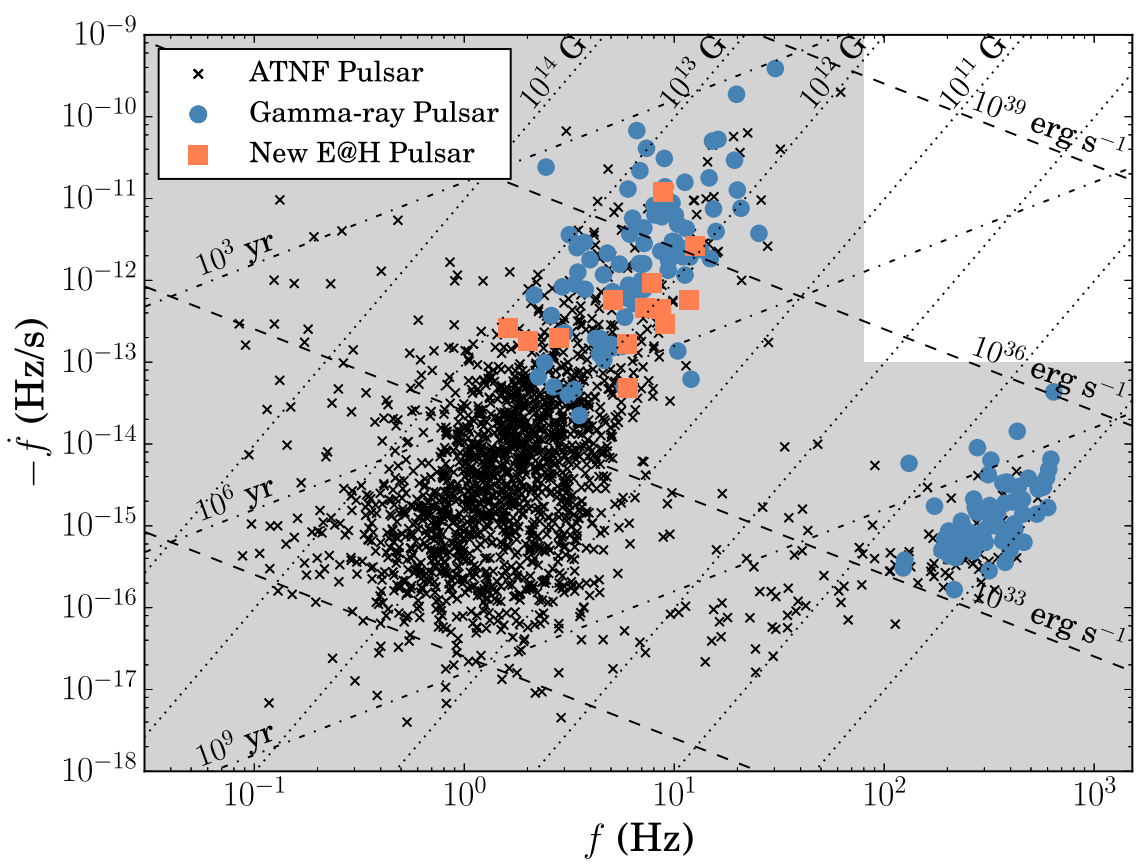

Figure 1. Frequency-spin-down diagram, showing the locations of non-gamma-ray pulsars in the ATNF Pulsar Catalogue (black crosses, Manchester et al. 2005), gamma-ray pulsars detected by Fermi-LAT (blue circles), and the newly detected Einstein@ Home pulsars reported in this work (orange squares). The parameter space covered by the Einstein @Home survey is shown by the gray shaded area. Lines of constant characteristic age, $\tau_{\mathrm{c}}=-f / 2 \dot{f}$ (dotted-dashed), surface magnetic field strength, $B_{\mathrm{S}}=\left(-1.5 I c^{3} \dot{f} f^{-3}\right)^{1 / 2} / 2 \pi R_{\mathrm{S}}^{3}$, (dotted) and spin-down power, $\dot{E}=-4 \pi^{2} I f \dot{f}$ (dashed) are also shown. To calculate these, we assumed neutron star moments of inertia, $I=10^{45} \mathrm{~g} \mathrm{~cm}^{2}$ and radii, $R_{\mathrm{S}}=10 \mathrm{~km}$, as in, e.g., Abdo et al. (2013). The timing analyses performed on the newly discovered pulsars, the results from which were used to calculate the properties above, are described in Section 4.

Some pulsars (e.g., PSRs J2017+3625 and J1350-6225) were found near the edge, or even slightly outside of their search regions, indicating that the confidence regions may be underestimated, and pulsars may have been missed by our survey as a result. This could be due to nearby, unmodeled gamma-ray sources "pulling" the apparent position of the source away from its true position, as was seen with PSR J1906 +0722 (Clark et al. 2015). To mitigate this effect in future surveys it may be necessary to search over larger regions, especially for sources at low Galactic latitude, where source confusion is more likely. However, increasing the solid angle over which we search increases the computational cost of the search by the same factor.

We split the search parameter space into two main regions: the young pulsar region, with spin frequencies below $80 \mathrm{~Hz}$, and the MSP region at higher spin frequencies. This parameter space is shown by the shaded area in Figure 1, and covers all currently known young pulsars, MSPs, and magnetars. In the low-frequency region, we extend the $\dot{f}$ range from 0 down to $-10^{-9} \mathrm{~Hz} \mathrm{~s}^{-1}$ to be sensitive to the youngest and most energetic pulsars. Older, recycled MSPs have much lower spin-down rates, and we therefore only search from 0 down to $-10^{-13} \mathrm{~Hz} \mathrm{~s}^{-1}$ in this region. Since more sky locations must be searched at higher frequencies, the majority of the computational cost of the search is spent in the high-frequency and high-spin-down regions. Pulsars whose pulse profile features two similarly sized peaks separated by half a rotation have most power in the second harmonic of their spin frequency. For this reason, we search up to $1520 \mathrm{~Hz}$, more than twice the frequency of the fastest known MSP, $716 \mathrm{~Hz}$ (Hessels et al. 2006). Only one known pulsar, PSR J0537 -6910 , has its second spin harmonic outside our parameter space (Marshall et al. 1998).
For each of the 118 unidentified LAT sources in which we searched, this parameter space is split into $\sim 10^{3}-10^{6}$ smaller work units, each of which can be searched in a few hours on a typical home computer. These work units are then distributed among Einstein@Home volunteers' computers.

\subsection{Detection Statistics}

In all stages of a gamma-ray pulsar search, statistical tests are used to measure the strength of pulsations for given rotational parameters. The detection statistics used in this survey are described in detail in Pletsch \& Clark (2014), and briefly defined here.

Kerr (2011) demonstrated the advantages of applying a weight to each photon indicating its probability of having come from the target source. The photon probability weights mentioned in Section 2.1, denoted by $\left\{w_{j}\right\}$, were therefore used to weight the contributions of each photon to a detection statistic. Weighting photons improves the sensitivity of a blind search by avoiding the need to apply specific photon energy and angular offset cuts, and by increasing the apparent fraction of flux that is pulsed.

To mitigate the computational cost of a blind search, semicoherent methods can be used, in which only photons arriving within a certain time difference from one another are combined coherently. The (real-valued) semicoherent detection statistic used in this search is defined as ${ }^{12}$

$$
S_{1}=\frac{1}{\kappa_{S_{1}}} \sum_{j=1}^{N} \sum_{k \neq j}^{N} w_{j} w_{k} e^{-i\left[\Phi\left(t_{j}\right)-\Phi\left(t_{k}\right)\right]} \hat{W}_{T}^{\mathrm{rect}}\left(\tau_{j k}\right)
$$

\footnotetext{
12 The subscript 1 here denotes that the detection statistic only sums power in the fundamental harmonic.
} 
where $\tau_{j k}$ is the time difference, or lag, between the arrivals of the $j$ th and $k$ th photons, and $\hat{W}_{T}^{\text {rect }}$ is a rectangular window, of length $T$,

$$
\hat{W}_{T}^{\text {rect }}(\tau)= \begin{cases}1, & |\tau| \leqslant T / 2 \\ 0, & \text { otherwise. }\end{cases}
$$

The $\kappa_{S_{1}}$ term of Equation (2) is a normalizing factor,

$$
\kappa_{S_{1}}=\sqrt{\sum_{j=1}^{N} \sum_{k \neq j}^{N} w_{j}^{2} w_{k}^{2} \hat{W}_{T}^{\text {rect }}\left(\tau_{j k}\right)},
$$

making the noise distribution of $S_{1}$ well approximated by a normal distribution with zero mean and unit variance.

The lag window length, $T$, is an important tuneable parameter for a search based on semicoherent methods. A longer lag window offers more sensitivity, but requires a finer grid in all four search parameters, and therefore results in a more costly search.

In the case where the lag window covers the entire observation span, all photons are combined fully coherently, and the test statistic reduces to the well-known Rayleigh test (modulo a constant term, and normalization) at the fundamental harmonic, $n=1$,

$$
\mathcal{P}_{n}=\frac{1}{\kappa^{2}}\left|\sum_{j=1}^{N} w_{j} e^{-i n \Phi\left(t_{j}\right)}\right|^{2},
$$

with the normalization constant,

$$
\kappa^{2}=\frac{1}{2} \sum_{j=1}^{N} w_{j}^{2}
$$

This is hereafter referred to as the coherent Fourier power at the $n$th harmonic.

To gain further sensitivity to weak signals, one can also combine the coherent Fourier power from several harmonics of the fundamental spin frequency. The well-known $H$-test developed by de Jager et al. (1989) offers a heuristic method for combining these harmonics in the typical case where the pulsar's pulse profile (and hence the distribution of Fourier power among the different harmonics) is unknown in advance by maximizing over the number of included harmonics, $M$, via

$$
H=\max _{1 \leqslant M \leqslant 20}\left(\sum_{n=1}^{M} \mathcal{P}_{n}-4 M+4\right) .
$$

Combining Fourier power from higher harmonics requires finer resolution in all phase model parameters. It therefore only becomes feasible in later search stages, in which the parameter space within which a candidate signal could lie is constrained to be very narrow.

As discussed in Pletsch \& Clark (2014), a multistage search scheme can be used to combine the efficiency of a semicoherent search with the superior sensitivity of fully coherent methods. In this scheme, the majority of the search is spent scanning the entire parameter space with the most efficient method available, before "following-up" the most interesting candidates in more sensitive stages.

\subsection{Initial Search Stage}

In this survey, the first stage used the semicoherent detection statistic, $S_{1}$, with a lag window of length $T=2^{21} \mathrm{~s} \approx 24$ days. This lag window is a factor of two longer than in previous Einstein@Home searches (Pletsch et al. 2013).

As described in Pletsch \& Clark (2014), the semicoherent detection statistic, $S_{1}$, defined in Equation (2), can be approximated more efficiently as a discrete Fourier transform (DFT), by utilizing the FFTW fast Fourier transform (FFT) algorithms (Frigo \& Johnson 2005). We hereby refer to the DFT form of the semicoherent detection statistic as $\hat{S}_{1}$.

Each FFT searched over a frequency bandwidth of $\Delta f_{\mathrm{BW}}=32 \mathrm{~Hz}$. We applied the technique of complex heterodyning, i.e., multiplying the FFT input vector by an additional sine wave at the heterodyning frequency, $f_{\mathrm{H}}$, to shift the search band to higher frequencies, $\left[f_{\mathrm{H}}-16, f_{\mathrm{H}}+16\right) \mathrm{Hz}$, without increasing the FFT memory size, $\Delta f_{\mathrm{BW}} T=256 \mathrm{MiB}$. This allows us to search for high-frequency signals, such as those from MSPs, using typical computing hardware. Furthermore, since the required resolution in the sky position becomes finer at higher frequencies, we can construct individual sky grids for each frequency band to avoid oversampling sky positions at low frequencies. The first frequency band was centered at $0 \mathrm{~Hz}$, and all frequencies below $5 \mathrm{mHz}$ were ignored to prevent harmonics of Fermi's orbital frequency $(\sim 0.175 \mathrm{mHz})$ from "drowning out" any astrophysical signal.

To ensure approximately equal sensitivity throughout the frequency band, we performed lag-domain interpolation (Pletsch \& Clark 2014), whereby each photon pair is interpolated into the 15 nearest bins on either side in the lagseries using a Welch-windowed sinc kernel (Welch 1967; Lyons 2004). Since this technique introduces an additional computational cost per pair of photons, we performed a photon weight cutoff to include at most the 30,000 highest-weight photons, ensuring that the FFT computation time remained the dominant factor. Identifying the photon weights as the probability of each photon being from a pulsar, the maximum (coherent) $\mathrm{S} / \mathrm{N}$ is proportional to $\sum_{j=1}^{N} w_{j}^{2}$. For sources where fewer than 30,000 photons were required to reach $95 \%$ of this maximum (typically sources far from the Galactic plane, where the diffuse background is lower), we increased the number of interpolation bins, up to a maximum of 30 .

A signal whose parameters, denoted by the vector $\boldsymbol{u}$, lie within the search space will, in general, not lie exactly at one of our search-grid points, and some of the $\mathrm{S} / \mathrm{N}$ is lost as a result of this offset, $\Delta \boldsymbol{u}$. We call this (fractional) loss in $\mathrm{S} / \mathrm{N}$ mismatch,

$$
m=1-\frac{\hat{S}_{1}(\boldsymbol{u}+\Delta \boldsymbol{u})}{\hat{S}_{1}(\boldsymbol{u})} .
$$

We can predict the expected mismatch as a function of the distance to the nearest search-grid point using an analytical "metric" approximation, as described in Pletsch \& Clark (2014). This prediction can then be used to construct an efficiently spaced grid of points in the parameter space at which to test for pulsations.

The spacing of frequency trials is fixed by the DFT formulation of $\hat{S}_{1}$ to be

$$
\Delta f=\frac{1}{T}
$$


While this spacing would result in a large average mismatch, we can improve upon this by performing simple "interbinning" (van der Klis 1989, pp. 27-69; Astone et al. 2010) to partially recover the lost $\mathrm{S} / \mathrm{N}$ experienced by signals lying between our frequency bins. This technique does not recover the full $\mathrm{S} / \mathrm{N}$ for such signals, but is far more efficient than the alternative of "zero-padding" the FFT to double length.

In the remaining parameters, we construct a cubic lattice with spacings chosen to provide the optimal maximum mismatch in each parameter of $m=0.15$ according to the metric approximation. In $\dot{f}$, the spacing depends on the lag window $T$ but also requires a refinement based on the full data set (Pletsch \& Allen 2009),

$$
\Delta \dot{f}=\frac{12 \sqrt{10 m}}{\pi T^{2}}\left[1+\frac{60}{N} \sum_{j=1}^{N} \frac{\left(t_{j}-t_{\mathrm{ref}}\right)^{2}}{T^{2}}\right]^{-1 / 2} .
$$

The grid of sky locations is first defined within a circle (with unit radius) in the ecliptic plane as a square grid with spacings in each direction of

$$
\Delta n_{x}=\Delta n_{y}=\frac{2 \sqrt{m}}{\pi f_{\max } r_{\mathrm{E}}}\left[1-\operatorname{sinc}^{2}\left(\Omega_{\mathrm{E}} T / 2\right)\right]^{-1 / 2},
$$

where $f_{\max }$ is the maximum frequency in the searched frequency band, $r_{\mathrm{E}}$ and $\Omega_{\mathrm{E}}$ are the Earth's orbital semimajor axis (in light seconds) and orbital angular frequency, respectively, and $\operatorname{sinc}(z)=\sin (z) / z$. These locations are then projected back into the celestial sphere to cover the LAT source localization region. At each location, barycentering corrections are applied to each photon's arrival time according to the JPL DE405 solar system ephemeris.

Each work unit performs an FFT at every location in this cubic lattice within its assigned portion of the parameter space. The five highest values of $\hat{S}_{1}$ (including interbinned samples) are stored in a running short list that is updated after each FFT. At the end of the semicoherent stage, this short list is saved, and each short-listed candidate is automatically "followed up" in additional, more sensitive search stages.

\subsection{Follow-up and Refinement Stages}

After the semicoherent stage, we are left with a small number of candidates in each work unit that have been localized to a small region of the parameter space. However, due to the large number of work units for each Fermi-LAT source, weak signals in these short lists can be of low overall significance. To separate weak signals from noise candidates, we can carry out more sensitive follow-up stages to act as a veto for the large number of candidate signals reported back by the semicoherent stage.

In the Einstein@Home survey, we implemented an intermediate refinement stage, in which candidates from the first stage are refined using a double-length lag window $\left(T=2^{22} \mathrm{~s} \approx 48\right.$ days). This step is computationally cheap, and narrows down the volume in which the candidate signal lies by a factor of $\sim 16$.

Following the semicoherent refinement stage, we now have a parameter space volume around each candidate that is small enough for a fully coherent search to be feasible with just a small associated computing cost. For this stage, we search only in the fundamental harmonic using the $\mathcal{P}_{1}$ test, with grid spacings according to the coherent metric approximation derived in Pletsch \& Clark (2014).

All search stages up to this point are carried out on the Einstein@Home volunteers' computers, after which the shortlisted candidates from the initial semicoherent stage (each of which were followed up), and the top candidates from the coherent follow-up stage are sent back to our servers.

As results are sent back, we update the top 20 most significant coherent candidates (see Appendix A for a description of the ranking procedure) overall from each source, and perform further follow-up and refinement procedures on them. First, we refine the location of the candidate using the $\mathcal{P}_{1}$ statistic, but calculated over a grid with a smaller mismatch $(m=0.05)$ than that used in the third stage. We then perform a fully coherent search using the $H$-test to incoherently sum the Fourier power in the first five harmonics.

After this refinement step, diagnostic plots for each candidate are produced that illustrate the candidates' signals and their evolution throughout the Fermi-LAT observation time. This allows us to identify pulsars with timing noise, whose pulsations may be visible in these plots despite having a low apparent coherent power due to variations in their signal phase.

\section{SENSITIVITY}

In Pletsch \& Clark (2014), the sensitivity of a blind search for gamma-ray pulsations was defined as the minimum pulsed fraction of the observed photon flux that can be detected with a fixed probability, $P_{\text {det }}^{*}$, and with a fixed false-alarm probability, $P_{\mathrm{FA}}^{*}$. We now apply this definition to investigate the sensitivity of our search to each source in the survey.

The quantity of interest is the fraction of the backgroundsubtracted weighted photon flux that is pulsed, denoted $p_{s}$. Given a set of photon weights, the fractions of the weighted photon counts that can be attributed to the source, $s$, and background fraction $b$, are estimated as (Guillemot et al. 2012; Abdo et al. 2013)

$$
s=\frac{\sum_{j=1}^{N} w_{j}^{2}}{\sum_{j=1}^{N} w_{j}}, \quad b=1-s .
$$

The probability of the $j$ th photon being pulsed is $w_{j} p_{s}$, and the overall weighted pulse profile takes the form

$$
g(\Phi)=\frac{b}{2 \pi}+s g_{s}(\Phi),
$$

where $g_{s}(\Phi)$ is the background-subtracted pulse profile,

$$
g_{s}(\Phi)=\frac{1-p_{s}}{2 \pi}+p_{s} g_{p}(\Phi)
$$

where $g_{p}(\Phi)$ is the pulse profile after subtracting all unpulsed emission (background or otherwise). These quantities are illustrated in Figure 2.

Note that this definition of the pulsed fraction is equal to the area under the pulse in the normalized pulse profile, as opposed to the "rms pulsed flux" used by e.g., Dib et al. (2009), which is additionally dependent on the shape of the pulse profile. While the rms pulsed flux provides a measure of the power of pulsations, this does not provide a physically meaningful measure of the proportion of pulsed flux emitted by the pulsar (Zhu et al. 2008).

For a pulsar to be detected by this survey, its signal must be strong enough to enter the short list of semicoherent candidates 


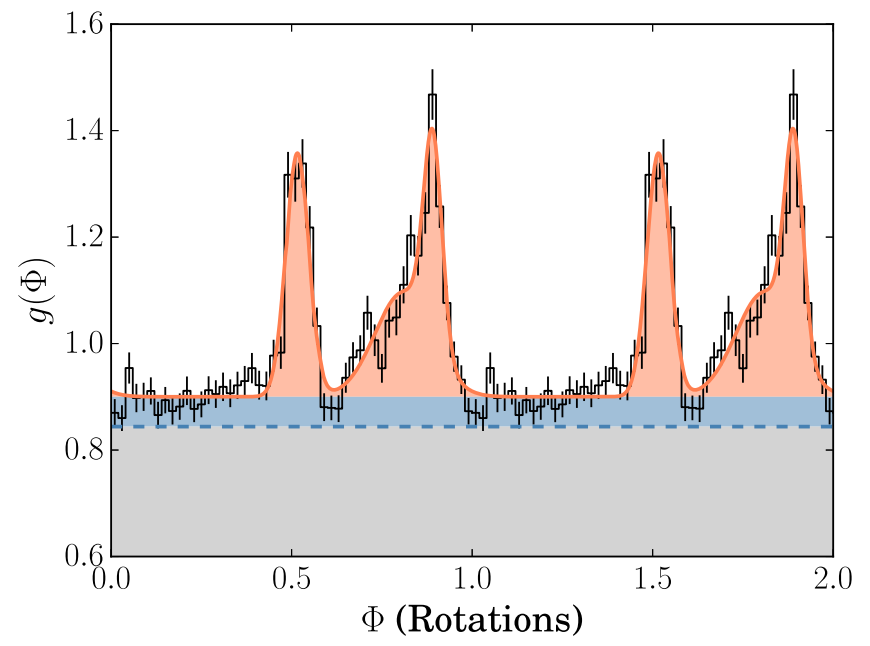

Figure 2. Gamma-ray pulse profile of PSR J2017+3625 illustrating the definition of the pulsed fraction. The blue dashed line indicates the background level, $b$. The pulsed fraction is defined by the area under the template pulse profile above its lowest level (the orange shaded area), divided by the source fraction, $s$ (that is the sum of blue and orange areas).

within the work unit that covers the region of the parameter space in which the signal lies. That is, the measured value of $\hat{S}_{1}$ at the grid point nearest the signal's location in the parameter space must be greater than the lowest value in the short list of candidates, $\hat{S}_{1}^{*}$. The probability that a signal with a pulse profile described by the complex Fourier coefficients $\left\{\gamma_{n}\right\}$ will be detected by the survey, as a function of the pulsed fraction is

$$
P_{\operatorname{det}}\left(p_{s} \mid\left\{\gamma_{n}\right\}\right)=\int_{-\infty}^{\infty} P\left(\hat{S}_{1}^{*}<\hat{S}_{1}\right) p\left(\hat{S}_{1} \mid p_{\mathrm{s}},\left\{\gamma_{n}\right\}\right) d \hat{S}_{1},
$$

where $P\left(\hat{S}_{1}^{*}<\hat{S}_{1}\right)$ is the (empirically measured) probability that $\hat{S}_{1}^{*}$ is less than $\hat{S}_{1}$, and $p\left(\hat{S}_{1} \mid p_{\mathrm{s}},\left\{\gamma_{n}\right\}\right)$ is the probability density function (pdf) of the measured value of $\hat{S}_{1}$ for a signal at a random location within the searched parameter space (see Appendix B for the derivation of this distribution). Each of these quantities depends additionally on the set of photon weights for each source; we have omitted these dependencies from Equation (15) for readability. This definition of the detection probability is illustrated in Figure 3. This equation can be solved numerically to recover the minimum pulsed fraction, $p_{\mathrm{s}}^{*}$, that can be detected at a given probability.

The purpose of the coherent follow-up stage of the survey is to greatly improve the significance of any true signal that is detected by the first stage, and we apply our final false-alarm probability threshold to the candidates from this stage. For typical values of $p_{s}$ given by solving Equation (15), the expected coherent power corresponds to an extremely significant signal $\left(\mathcal{P}_{1} \approx 270, P_{\mathrm{FA}} \sim 10^{-59}\right)$ and hence even a conservative false-alarm threshold has no real effect on the overall sensitivity estimate. In practice, effects such as glitches or timing noise that are not included in our simplified isolated pulsar phase model can severely reduce the observed coherent power, resulting in true signals with large $p_{s}$ appearing with low significance. We attempt to mitigate these effects somewhat by monitoring the 20 most significant candidates from both the semicoherent stage and the follow-up stages rather than applying the false-alarm threshold rigorously.

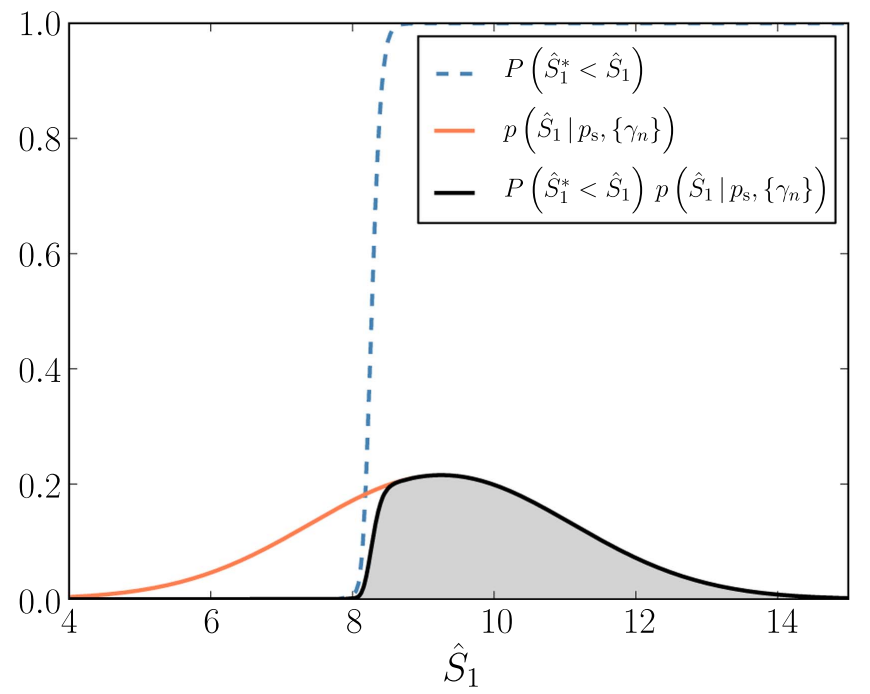

Figure 3. Illustration of the definition of the detection probability. The orange line indicates the pdf of the semicoherent detection statistic (including mismatch) in the presence of a signal with fixed pulse profile and pulsed fraction. The blue dashed line shows the empirical probability that a true signal resulting in a detection statistic $\hat{S}_{1}$ will be followed up (and hence detected). The detection probability is therefore the area under the product of these functions, shown by the gray shaded area.

In Table 1, we estimate the minimum pulsed fraction, $p_{s}^{*}$, that can be detected with $P_{\text {det }}^{*}=0.95$ for each source in our survey, averaged over the pulse profiles from the 30 most significant pulsars in the $2 \mathrm{PC}$, and assuming constant signal parameters (i.e., no glitches or significant timing noise). For sources in which a new gamma-ray pulsar was discovered, we also report the measured pulsed fraction for illustration. Note that while some sources have $p_{s}^{*}>1$, this does not necessarily preclude the detection of pulsed emission from this source, since the assumptions on which these estimates are based might not always apply. For example, the true pulse profile could be narrower than average or the photon weights might not accurately represent the probability of each photon coming from the target source. The results of this sensitivity estimation displayed in Figure 4, therefore, also illustrate the range of thresholds covered by the various pulse profiles observed.

While the semicoherent search stage is rather robust to the presence of timing noise, any large enough deviation from our simplified constant spin-down model will significantly affect the sensitivity of our search. Glitching pulsars, for example, are particularly difficult to detect because their large jumps in spin frequency prevent the $\mathrm{S} / \mathrm{N}$ from accumulating steadily throughout the observations. Pulsars in binary systems are all but impossible to find in a search for isolated pulsars. Our pulsed fraction thresholds, therefore, only represent our sensitivity to well-behaved isolated pulsars. In particular, our sensitivity estimates are likely to be most reliable for sources far from the Galactic plane, where we would expect to find old, stable MSPs. Our sensitivity estimates rely on the assumption that a pulsar lies within the sky region in which we search. As mentioned in Section 2.2, in some cases, the extension of this region may have been underestimated.

We have also not considered the fact that our sensitivity is not exactly constant throughout the parameter space. Rather, the grid of sky locations slightly overcovers the region at low frequencies and, due to the lower number of sky points required 
Table 1

Pulsed Fraction Upper Limits

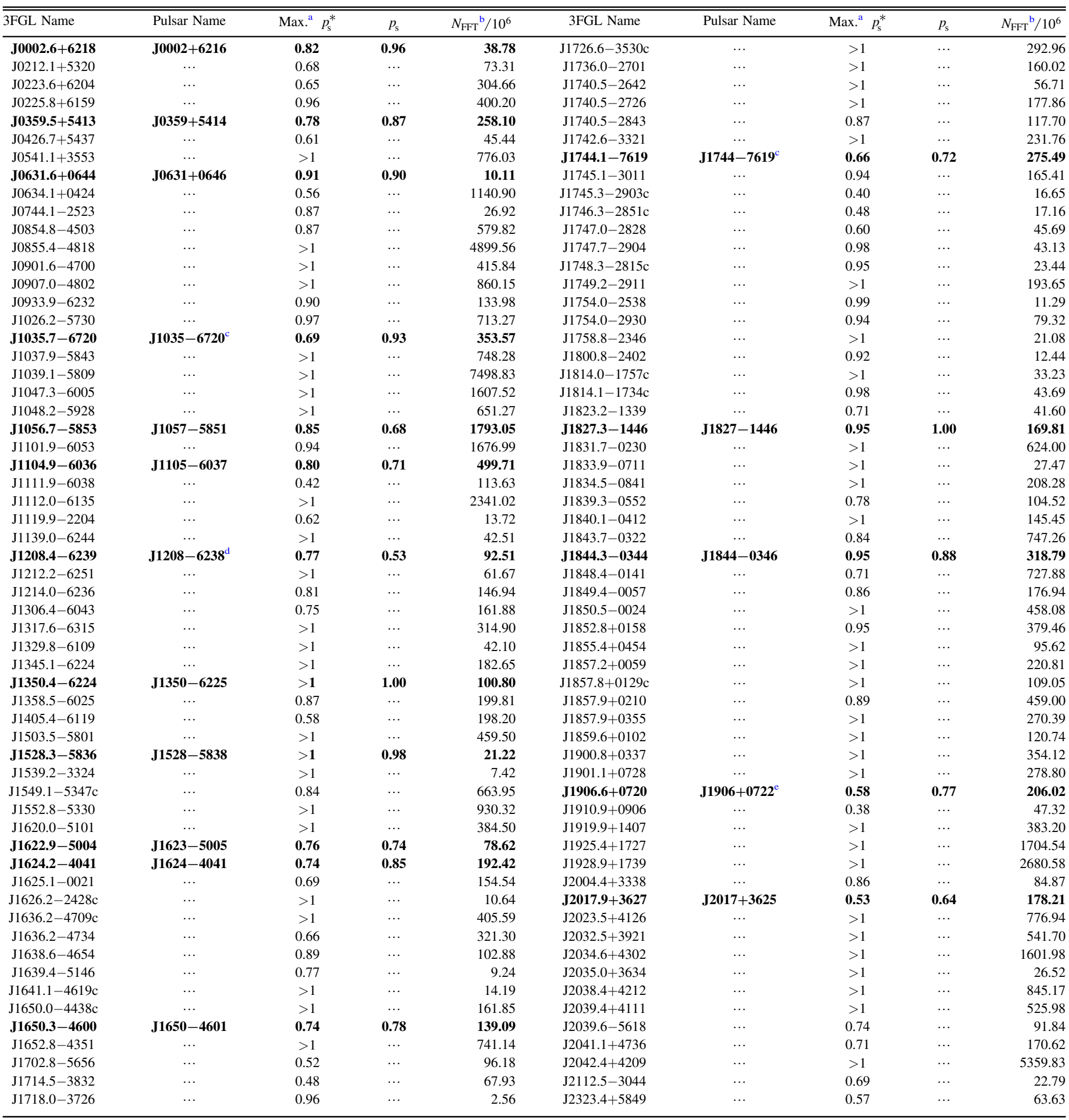

Notes. Sources in which pulsars were discovered by the Einstein@Home survey are shown in bold. For some of these pulsars, the measured pulsed fraction is well below our estimated upper limit. This can be due to the pulsar having a narrower-than-average pulse profile, a very low spin frequency (at which the sky grid, constructed for the highest frequency in the search band, greatly overcovers the search region), or simple "luck" in that the signal lies close to one of our search points and has a lower-than-average mismatch. The $95 \%$ detection probability requirement therefore results in conservative limits.

${ }^{\text {a }}$ Estimated values for the pulsed fraction above which we expect to detect a signal from each source with $95 \%$ probability.

${ }^{\mathrm{b}}$ Number of FFTs required to search the entire parameter space for each source.

${ }^{c}$ These pulsars have timing properties warranting further individual investigation and will be presented in later works.

d The discovery and analysis of PSR J1208-6238 was presented in Clark et al. (2016).

${ }^{\mathrm{e}}$ The discovery and analysis of PSR J1906+0722 was presented in Clark et al. (2015). 


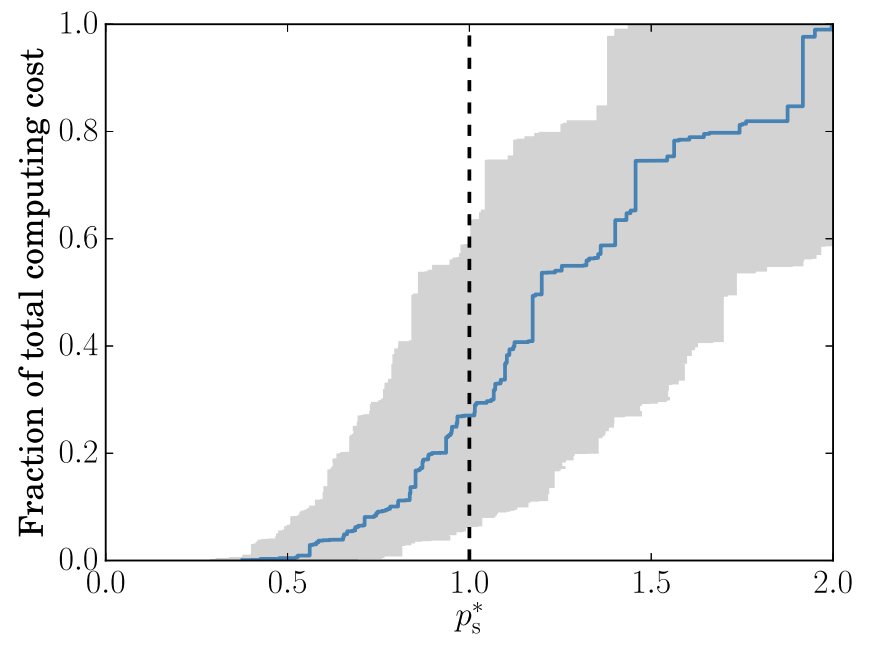

Figure 4. Cumulative fraction of the total computing cost of the survey, as a function of the estimated pulsed fraction threshold. The solid line shows the $95 \%$ detection probability pulsed fraction threshold averaged over the pulse profiles of the 30 pulsars in the $2 \mathrm{PC}$ with the highest significance. The shaded area shows the range of pulsed fraction thresholds over these pulse profiles. The dashed vertical line denotes the maximum expected pulsed fraction of $p_{\mathrm{s}}^{*}=1$.

at low frequencies, work units searching the lowest frequency band often search at a smaller number of trials. The result is that our survey is sometimes slightly more sensitive at low spin frequencies. The results of Table 1 have been averaged over all spin frequencies, and assume that the entire parameter space is equally well-covered by search points.

In the following sections, we highlight and discuss the implications of our measured pulsed fraction upper limits for three sources with well-known counterparts from observations at other wavelengths, from which no pulsations were detected by our survey.

\subsection{Pulsed Fraction Upper Limit for W49B}

Chandra observations of the supernova remnant (SNR) W49B (3FGL J1910.9+0906), believed to be the remnant of a jet-driven, core-collapse supernova, place strong upper limits on the presence of a neutron star (Lopez et al. 2013).

Gamma-rays from W49B have been detected at energies far higher than observed from a typical gamma-ray pulsar (Abdo et al. 2010b; H.E.S.S. Collaboration 2016), indicating that the majority of the high-energy flux from W49B comes from the SNR itself. Any pulsed emission from a gamma-ray pulsar would, therefore, likely represent only a small fraction of the photon flux. Our results place a $95 \%$ pulsed fraction limit of $38 \%$ of the weighted photon flux from this source.

Placing W49B at the distance of $10 \mathrm{kpc}$ obtained by Zhu et al. (2014) suggests a gamma-ray luminosity of $\sim 2 \times 10^{36}$ erg $\mathrm{s}^{-1}$. In order to provide a significant fraction of this emission, any gamma-ray pulsar would have to have a very large spin-down power, since the efficiency of converting spindown power into gamma-ray luminosity tends to be lower for energetic pulsars (Abdo et al. 2013). The estimated age of the SNR is in the range of 1000-4000 years. Together, these observations suggest that any potential gamma-ray pulsar would be very young and extremely energetic, and would likely exhibit large timing noise and glitches as a result. This would seriously reduce the sensitivity of our blind search of this target, making our upper limit estimate for the pulsed flux unreliable for this source.
To check for signals with large timing noise, we manually followed up semicoherent candidates from this source using refinement steps with increasing lag-window lengths, but none revealed a significant pulsed signal.

\subsection{Pulsed Fraction Upper Limit for Cassiopeia A}

The SNR Cassiopeia A (Cas A) contains a point-like, central X-ray source, most likely a neutron star (Ho \& Heinke 2009), from which no pulsations have been detected in X-rays, gamma-rays, or radio observations. The gamma-ray spectrum for this source is also unlike that of any pulsar, again suggesting that any pulsed emission would likely only contribute a fraction of the total observed flux. The position of this central compact object is within our search region for 3FGL J2323.4+5849, a source for which our survey sets a pulsed fraction upper limit of $p_{s}^{*}=57 \%$. The photon flux above $100 \mathrm{MeV}$ from this source was $3.1(2) \times 10^{-8}$ photons $\mathrm{cm}^{-2} \mathrm{~s}^{-1}$, making our $95 \%$ upper limit more than an order of magnitude lower than the $5 \sigma$ limit for pulsed flux reported in Abdo et al. (2010a). A dedicated search for pulsations at the known position of the Cas A neutron star, excluding photons above typical pulsar emission energies, could likely bring this limit down further.

However, since Cas A is known to be a young SNR (the supernova occurred around A.D. 1680), if the NS is indeed a pulsar, it will be very energetic and likely have a highly unstable spin, making detection in a blind search extremely challenging even if the pulsed fraction is far higher than our stated upper limit. Indeed, the SNR is young enough that the pulsar's spin-down could even be outside our search range (Abadie et al. 2010). Again, we followed up semicoherent candidates from this source, without success.

\subsection{Pulsed Fraction Upper Limit for the Galactic Center}

As a result of intense and difficult-to-model interstellar emission, the area around the Galactic Center (GC) is one of the most complicated, and hence poorly understood regions of gamma-ray emission. Both the 3FGL and the recent First Fermi-LAT Inner Galaxy Point Source Catalog (Ajello et al. 2016) identify several bright point sources within a few degrees of the GC; though, some of these could be due to misattributed interstellar emission. Nevertheless, a substantial contribution to the flux from the GC region is expected to come from other astrophysical sources, such as young or MSPs (e.g., Bartels et al. 2016; O'Leary et al. 2016, submitted, and references therein), or possibly even annihilating dark matter particles (Hooper et al. 2013, and references therein). The detection of a gamma-ray pulsar near the GC would have important implications for these two competing interpretations of the GC GeV flux.

The bright fore-/background from the interstellar medium makes blindly searching for pulsars near the GC particularly difficult. In order for one single pulsar to be detectable above this background, it must be extremely bright, especially if it lies at a similar distance as the GC, $\sim 8 \mathrm{kpc}$. As an example, the bright source 3FGL J1745.3-2903c searched during this survey has $p_{\mathrm{s}}^{*}=40 \%$, corresponding to a pulsed photon flux above $1 \mathrm{GeV}$ of $\sim 10^{-8}$ photons $\mathrm{cm}^{-2} \mathrm{~s}^{-1}$. This flux is similar to the photon flux that the Crab pulsar would produce if it was at the same distance as the GC. Again, such highly luminous pulsars also exhibit the most timing noise and glitches, further 
adding to the difficulty of detecting their pulsations above the bright background flux.

\section{TIMING ANALYSIS}

We performed timing analyses for each new pulsar to precisely determine their sky positions and rotational parameters, again denoted by the vector $\boldsymbol{u}$. The analysis follows the procedure described in Clark et al. (2015), as an extension of the method described by Ray et al. (2011).

For the purpose of these follow-up timing analyses, we produced extended LAT data sets until 2015 September 9 for each of the pulsars newly reported in this work. These updated data sets were produced using the P8R2_SOURCE_V6 IRFs, gll_iem_v06.fits Galactic diffuse emission template (Acero et al. 2016), and iso_P8R2_SOURCE_V6_v06.txt isotropic diffuse background template. ${ }^{13}$ The extended data sets had a lower zenith angle cutoff of $90^{\circ}$. The pulsar's position was fixed at its initial timing position. Photons from within a larger $15^{\circ}$ radius were included in the likelihood fitting, which was performed using gtlike. Photon weights were then calculated for all photons from within $5^{\circ}$ of the pulsar using gtsrcprob. Further details of the preparation of these data will be given in Paper II, including the spectral properties of each newly detected pulsar.

To reduce the number of photons included in the timing analysis for computational efficiency, we applied a photon weight cutoff with the minimum photon weight chosen such that no more than $1 \%$ of the maximum coherent Fourier power was lost (again assuming that the maximum coherent $\mathrm{S} / \mathrm{N}$ is proportional to $\sum_{j=1}^{N} w_{j}^{2}$ ).

Starting from the spin and positional parameters of the pulsar reported by the refinement stage, we phase-folded the photon data to obtain a weighted pulse profile. We also phase-folded at half and one-third of the measured frequency to ensure that the original signal was not a higher harmonic of the fundamental spin frequency. In two pulsars, J1350-6225 and J1624-4041, this revealed sharply double-peaked profiles at half of the original candidate frequency, and greatly increased their measured $H$-test values, indicative of having identified the true spin frequency.

From the phase-folded data, we constructed a template pulse profile, $\hat{g}_{s}(\Phi)$, consisting of a combination of symmetrical wrapped Gaussian peaks (as defined in Abdo et al. 2013), which were fit by maximizing the likelihood,

$$
\mathcal{L}\left(\hat{g}_{s}, \boldsymbol{u}\right)=\prod_{j=1}^{N}\left[w_{j} \hat{g}_{s}\left(\Phi\left(t_{j}, \boldsymbol{u}\right)\right)+\left(1-w_{j}\right)\right] .
$$

The number of peaks in the template profile was chosen by the template that minimizes the Bayesian Information Criterion (BIC, Schwarz 1978),

$$
\mathrm{BIC}=-2 \log \left(\mathcal{L}\left(\hat{g}_{s}, \boldsymbol{u}\right)\right)+k \log \left(\sum_{j=1}^{N} w_{j}\right),
$$

where $k$ is the number of free parameters in the model. Because each Gaussian peak consists of three parameters (central phase, width, and amplitude), when fitting the template pulse profile, $k=3 N_{\text {peaks. }}$. Due to the presence of the second term in Equation (17), a new component was only added to the

\footnotetext{
13 http://fermi.gsfc.nasa.gov/ssc/data/access/lat/BackgroundModels.html
}

template profile if its presence significantly increased the likelihood. It therefore acts as a penalty factor, discriminating against a template profile featuring many "spiky" components, unless this is warranted by the data. The parameters of the template pulse profiles used to time each pulsar are given in Table 2, and the profiles themselves are shown in Figure 5.

After obtaining the template pulse profile, we varied the positional and spin parameters and explored the resulting multidimensional likelihood surface to find the most likely parameter values. To explore the likelihood surface, we used the Affine Invariant Monte Carlo method described by Goodman \& Weare (2010), in which many Monte Carlo chains are run in parallel, with proposal jumps for each chain depending on the locations of the other chains. We used the scheme described by Foreman-Mackey et al. (2013) to efficiently parallelize the likelihood computations among several CPU cores.

With the new parameter values, we re-folded the photon data to obtain a new template pulse profile as above. Additional parameters could then be added to the timing model, and the procedure was repeated. For each pulsar, we started from the simplified timing model (i.e., up to $\dot{f}$ ), added higher frequency derivative terms, found the most likely parameters, and updated the template pulse profile until the BIC of Equation (17) (with $k$ now as the number of parameters in the timing model) stopped decreasing. With the number of timing parameters selected in this manner, we performed a final longer Monte Carlo run, using a large number of chains, to obtain precise estimates of the mean value and uncertainty of each parameter.

With over 100,000 photons whose individual barycentric arrival times must be computed each time, each likelihood evaluation is relatively computationally expensive. Hence, efficient convergence of the Monte Carlo step is crucial to allow us to perform the timing analysis in a reasonable amount of time. To avoid the possibility of chains getting stuck in lowlikelihood regions, we start all of the chains in a tight ball near our current most-likely point, as advocated by ForemanMackey et al. (2013). Using a lengthy burn-in period, we allow these chains to spread out throughout the most likely regions of our parameter space. While this initialization can, in principle, lead to the Monte Carlo sampling only reaching a local likelihood maximum rather than exploring the full parameter space to find a global maximum, visual inspection of the phasefolded photon data can typically reveal any significant residuals in the timing solution requiring further fitting.

The results of these analyses are summarized in Table 3 . The physical properties of each of the new pulsars, as derived from their spin frequency and spin-down rate are given in Table 4.

These timing solutions allow for sensitive follow-up searches, the identification of candidate multiwavelength counterparts, and phase-resolved spectroscopy of the on- and off-pulse photons. Dedicated radio observations of the newly discovered pulsars were also performed, which used the gamma-ray timing solution to fold the data. For those pulsars that were subsequently detected in radio observations, the phase alignment between the radio and gamma-ray pulses provide constraints on the pulsars' emission geometry and inclination, allowing for the comparison of different gammaray emission models (e.g., Johnson et al. 2014). These analyses and their results will be described in Paper II. 
Table 2

Template Pulse Profile Parameters

\begin{tabular}{|c|c|c|c|c|c|c|c|c|c|c|c|}
\hline Pulsar & $a_{1}$ & $\sigma_{1}$ & $\Delta \mu_{2}$ & $a_{2}$ & $\sigma_{2}$ & $\Delta \mu_{3}$ & $a_{3}$ & $\sigma_{3}$ & $\Delta \mu_{4}$ & $a_{4}$ & $\sigma_{4}$ \\
\hline $\mathrm{J} 0002+6216$ & 0.32 & 0.088 & -2.476 & 0.25 & 0.186 & -0.319 & 0.26 & 0.217 & -1.531 & 0.68 & 0.697 \\
\hline $\mathrm{J} 0359+5414$ & 1.36 & 0.511 & $\ldots$ & $\ldots$ & $\ldots$ & $\ldots$ & $\ldots$ & $\ldots$ & $\ldots$ & $\ldots$ & $\ldots$ \\
\hline $\mathrm{J} 0631+0646$ & 0.29 & 0.145 & -1.821 & 0.62 & 0.374 & -0.663 & 0.50 & 0.408 & $\cdots$ & $\cdots$ & $\cdots$ \\
\hline $\mathrm{J} 1057-5851$ & 0.43 & 0.301 & -1.143 & 0.65 & 0.907 & $\ldots$ & $\ldots$ & $\ldots$ & $\ldots$ & $\ldots$ & $\ldots$ \\
\hline $\mathrm{J} 1105-6037$ & 0.19 & 0.116 & -2.026 & 0.46 & 0.294 & -0.632 & 0.47 & 0.866 & $\cdots$ & $\cdots$ & $\ldots$ \\
\hline $\mathrm{J} 1350-6225$ & 0.57 & 0.077 & 3.053 & 0.31 & 0.079 & 0.278 & 0.62 & 0.453 & $\cdots$ & $\cdots$ & $\cdots$ \\
\hline J1528-5838 & 0.13 & 0.053 & 0.972 & 1.42 & 0.762 & $\ldots$ & $\ldots$ & $\ldots$ & $\ldots$ & $\ldots$ & $\ldots$ \\
\hline $\mathrm{J} 1623-5005$ & 0.30 & 0.151 & -2.215 & 0.17 & 0.141 & -1.438 & 0.70 & 0.855 & $\ldots$ & $\ldots$ & $\ldots$ \\
\hline $\mathrm{J} 1624-4041$ & 0.31 & 0.080 & -2.715 & 0.11 & 0.075 & -2.328 & 0.50 & 0.383 & -0.072 & 0.42 & 0.604 \\
\hline $\mathrm{J} 1650-4601$ & 0.38 & 0.210 & 2.079 & 0.33 & 0.211 & 1.227 & 0.52 & 0.571 & $\ldots$ & $\cdots$ & $\ldots$ \\
\hline $\mathrm{J} 1827-1446$ & 1.44 & 0.311 & -1.581 & 0.13 & 0.182 & $\cdots$ & $\cdots$ & $\ldots$ & $\cdots$ & $\cdots$ & $\cdots$ \\
\hline $\mathrm{J} 1844-0346$ & 1.38 & 0.467 & $\cdots$ & $\cdots$ & $\cdots$ & $\cdots$ & $\cdots$ & $\cdots$ & $\cdots$ & $\cdots$ & $\cdots$ \\
\hline $\mathrm{J} 2017+3625$ & 0.37 & 0.200 & 2.356 & 0.25 & 0.160 & 1.843 & 0.39 & 0.494 & $\ldots$ & $\ldots$ & $\ldots$ \\
\hline
\end{tabular}

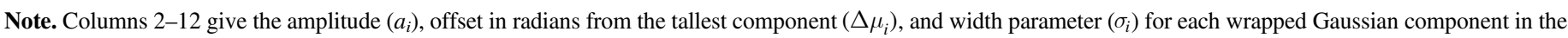
template pulse profile.

\subsection{Spin-down versus Timing Noise}

The long-term spin-down behavior of a pulsar can be characterized by the braking index (e.g., Lyne et al. 2015), n, where,

$$
\begin{gathered}
\dot{f} \propto-f^{n}, \\
n=\frac{f \ddot{f}}{\dot{f}^{2}} .
\end{gathered}
$$

The exact value of the braking index depends on the physical mechanism causing the pulsar to spin down; a pulsar whose braking is entirely due to magnetic dipole radiation will have $n=3$, whereas one whose spin-down power is entirely due to the radiation of gravitational waves will have $n=5$ or $n=7$ (Abadie et al. 2010).

The vast majority of pulsars, however, also exhibit redspectrum timing noise, manifesting as low-frequency quasiperiodic variations in the arrival times of pulses (e.g., Hobbs et al. 2010; Kerr et al. 2015). The amplitude of this timing noise appears to correlate with the spin-down energy, $\dot{E}$, which is typically higher for gamma-ray pulsars than the rest of the pulsar population. For all but the youngest pulsars or those with the highest magnetic fields, this timing noise obscures the true long timescale braking behavior.

In all pulsars measured here, $n$ deviates significantly from any of these integer values (except for PSR J1650-4601, where the index is low, but poorly constrained), suggesting that the measured values of $\ddot{f}$ are due to short timescale timing noise. For pulsars with measurable frequency derivative terms beyond the first derivative, the evolution of the spin frequency and spin-down rate is shown in Figure 6.

\subsection{Timing Large Glitches}

In addition to slowly varying timing noise behavior, young pulsars also occasionally exhibit large, sudden changes in their spin frequency, known as "glitches" (e.g., Yu et al. 2013). Pulsars with large glitches are particularly difficult to detect in blind searches, which require long intervals containing a stable signal to accumulate sufficient $\mathrm{S} / \mathrm{Ns}$.

Not only are pulsars with large glitches harder to detect in a blind search, they are also significantly more complicated to time. A glitch occurring at time $t_{\mathrm{g}}$, with increments in the spin frequency $\left(\Delta f_{\mathrm{g}}\right)$, the first two frequency derivatives $\left(\Delta \dot{f}_{\mathrm{g}}, \Delta \ddot{f}_{\mathrm{g}}\right)$ and an exponentially decaying frequency increment $\left(\Delta f_{\mathrm{D}, \mathrm{g}}\right.$, decay timescale $\tau_{\mathrm{D}, \mathrm{g}}$ ) causes a phase offset at time $t>t_{\mathrm{g}}$ of

$$
\begin{aligned}
\Delta \Phi_{\mathrm{g}}(t)= & 2 \pi\left[\Delta f_{\mathrm{g}}\left(t-t_{\mathrm{g}}\right)+\frac{\Delta \dot{f}_{\mathrm{g}}}{2}\left(t-t_{\mathrm{g}}\right)^{2}\right. \\
& +\frac{\Delta \ddot{f}_{\mathrm{g}}}{6}\left(t-t_{\mathrm{g}}\right)^{3} \\
& +\Delta f_{\mathrm{D}, \mathrm{g}} \tau_{\mathrm{D}, \mathrm{g}}\left(1-\mathrm{e}^{\left.-\left(t-t_{\mathrm{g}}\right) / \tau_{\mathrm{D}, \mathrm{g}}\right)}\right] .
\end{aligned}
$$

If the parameter increments associated with the glitch are large enough, then $\Delta \Phi_{\mathrm{g}}$ can quickly exceed a small integer number of rotations. If the photon data are sparse, as is often the case, this can happen before we have even detected any pulsed photons after the glitch (Yu et al. 2013; Clark et al. 2015).

A result is that the likelihood distribution in the glitch epoch, $t_{\mathrm{g}}$, resembles a comb of possible epochs, with each maximum occurring at a time where $\Delta \Phi_{\mathrm{g}}(t)$ equals an integer number of rotations. Such a highly multi-modal likelihood surface causes problems for our Monte Carlo sampling method, as chains can easily get stuck in low-likelihood regions between maxima, greatly reducing the efficiency of the sampling procedure.

To avoid this, we can include an arbitrary phase increment at the time of the glitch in our phase model and allow it to vary as part of the Monte Carlo sampling. This phase increment removes the multi-modal nature of the likelihood surface by accounting for the phase offset for glitch models that do not occur at one of the maxima described above. While the phase increment is highly correlated with the glitch epoch, we can predict and account for the dominant contribution to the time-dependent part of the glitch increment to remove this correlation, ensuring efficient sampling (see Appendix $\mathrm{C}$ for further details).

Apart from PSR J1906+0722, (published previously in Clark et al. 2015) one other pulsar detected by the Einstein@ Home survey, PSR J1844-0346, experienced a glitch during the Fermi mission. Occurring in 2012 July-August, with $\Delta f / f \approx 3.5 \times 10^{-6}$, it was comparable to some of the largest glitches detected from gamma-ray pulsars (Pletsch et al. 2012b). With a characteristic age of $\tau_{c}=11.6 \mathrm{kyr}$, and 

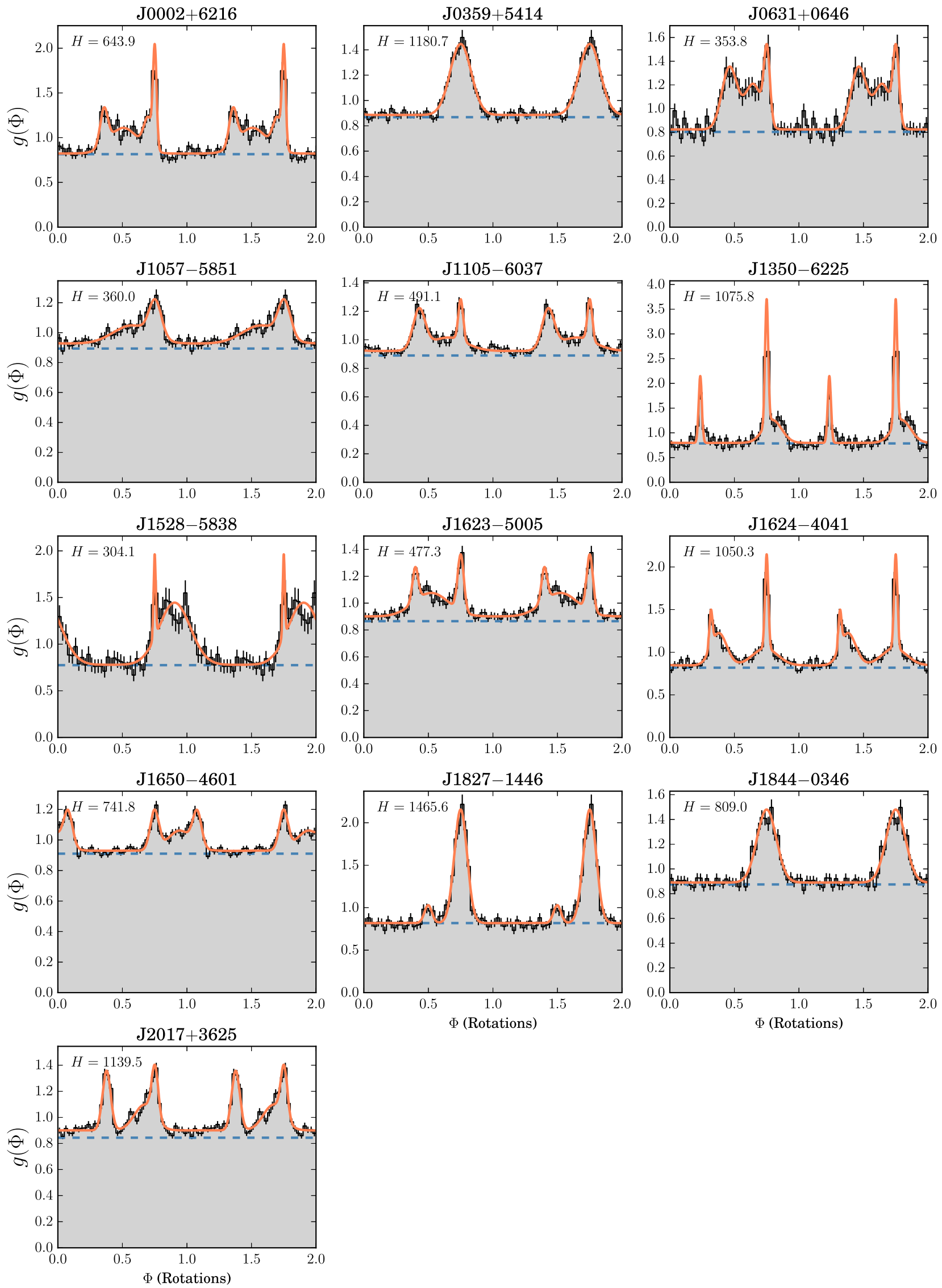

Figure 5. Weighted pulse profiles of all pulsars reported in this work. The template pulse profiles used for timing analyses are shown by orange curves. The background fraction is illustrated by the dashed blue line in each plot. 
Table 3

Pulsar Timing Parameters

\begin{tabular}{|c|c|c|c|c|c|}
\hline Pulsar & $t_{\mathrm{ref}}(\mathrm{MJD})$ & R.A. & Decl. & $f(\mathrm{~Hz})$ & $\dot{f}\left(10^{-12} \mathrm{~Hz} \mathrm{~s}^{-1}\right)$ \\
\hline $\mathrm{J} 0002+6216$ & 55806 & $00^{\mathrm{h}} 02^{\mathrm{m}} 58^{\mathrm{s}} .17(2)$ & $+62^{\circ} 16^{\prime} 09^{\prime \prime} 4(1)$ & $8.6682478274(1)$ & $-0.448354(5)$ \\
\hline $\mathrm{J} 0359+5414$ & 55716 & $03^{\mathrm{h}} 59^{\mathrm{m}} 26^{\mathrm{s}} .01(2)$ & $+54^{\circ} 14^{\prime} 55^{\prime \prime} 7(3)$ & $12.5901403227(2)$ & $-2.65247(1)$ \\
\hline J0631+0646 & 55806 & $06^{\mathrm{h}} 31^{\mathrm{m}} 52^{\mathrm{s}} \cdot 38(2)$ & $+06^{\circ} 46^{\prime} 14^{\prime \prime} 2(7)$ & $9.01071834910(6)$ & $-0.293694(2)$ \\
\hline $\mathrm{J} 1057-5851$ & 55716 & $10^{\mathrm{h}} 57^{\mathrm{m}} 09^{\mathrm{s}} \cdot 5(2)$ & $-58^{\circ} 51^{\prime} 07(2)^{\prime \prime}$ & $1.6119541713(3)$ & $-0.26135(1)$ \\
\hline J1105-6037 & 55716 & $11^{\mathrm{h}} 05^{\mathrm{m}} 00 \mathrm{~s} \cdot 48(4)$ & $-60^{\circ} 37^{\prime} 16^{\prime \prime} 3(3)$ & $5.12982912390(8)$ & $-0.574649(2)$ \\
\hline $\mathrm{J} 1350-6225$ & 55806 & $13^{\mathrm{h}} 50^{\mathrm{m}} 44^{\mathrm{s}} .45(1)$ & $-62^{\circ} 25^{\prime} 43^{\prime \prime} 8(1)$ & $7.23810134280(6)$ & $-0.465408(2)$ \\
\hline J1528-5838 & 55806 & $15^{\mathrm{h}} 28^{\mathrm{m}} 24^{\mathrm{s}} \cdot 3(1)$ & $-58^{\circ} 38^{\prime} 01(1)^{\prime \prime}$ & $2.81146362521(6)$ & $-0.195700(1)$ \\
\hline $\mathrm{J} 1623-5005$ & 55716 & $16^{\mathrm{h}} 23^{\mathrm{m}} 04^{\mathrm{s}} .11(1)$ & $-50^{\circ} 05^{\prime} 15^{\prime \prime} 1(2)$ & $11.7547287226(1)$ & $-0.574965(3)$ \\
\hline J1624-4041 & 55716 & $16^{\mathrm{h}} 24^{\mathrm{m}} 09^{\mathrm{s}} .927(9)$ & $-40^{\circ} 41^{\prime} 29^{\prime \prime} \cdot 7(3)$ & $5.95730476591(3)$ & $-0.1676839(9)$ \\
\hline J1650-4601 & 55716 & $16^{\mathrm{h}} 50^{\mathrm{m}} 18^{\mathrm{s}} \cdot 62(2)$ & $-46^{\circ} 01^{\prime} 18 . \prime 6(4)$ & $7.8664037135(1)$ & $-0.937157(3)$ \\
\hline J1827-1446 & 55716 & $18^{\mathrm{h}} 27^{\mathrm{m}} 24^{\mathrm{s}} .60(5)$ & $-14^{\circ} 46^{\prime} 28(4)^{\prime \prime}$ & $2.0032588600(1)$ & $-0.181932(3)$ \\
\hline J1844-0346 & 55716 & $18^{\mathrm{h}} 44^{\mathrm{m}} 32^{\mathrm{s}} .89(2)$ & $-03^{\circ} 46^{\prime} 30^{\prime \prime} 6(9)$ & $8.8609552273(8)$ & $-12.14675(5)$ \\
\hline $\mathrm{J} 2017+3625$ & 55716 & $20^{\mathrm{h}} 17^{\mathrm{m}} 55^{\mathrm{s}} \cdot 84(1)$ & $+36^{\circ} 25^{\prime} 07^{\prime \prime} \cdot 9(2)$ & $5.99703102436(3)$ & $-0.0489063(8)$ \\
\hline
\end{tabular}

Note. Reported values of timing parameters are the mean values obtained from the Monte-Carlo analysis described in Section 4 at the reference epoch, $t_{\text {ref }}$, with $1 \sigma$ uncertainties in the final digits given in brackets. Reference epochs are in Barycentric Dynamical Time (TDB). Two different observation spans were used during this survey and the reference epochs were chosen to lie at the middle of the observation, hence the two distinct values shown in column 2.

spin-down energy $\dot{E}=4.2 \times 10^{36} \mathrm{erg} \mathrm{s}^{-1}$, this pulsar is by far the most energetic pulsar found by our survey and also displays a correspondingly large degree of timing noise. The evolution of the spin frequency and spin-down rate of PSR J1844+0346, including the glitch, are shown in Figure 7. The glitch parameters obtained from the timing analysis are given in Table 5.

\subsection{Pulse Profile Variability}

We checked for variations in the pulse profiles of all pulsars detected in this survey by visually inspecting their phase-time diagrams, and by measuring their Fourier coefficients in a small number of time intervals. In one pulsar, PSR J1350-6225, small changes in the first and second Fourier coefficients were observed. This was also observed in the phase-time diagram for this pulsar (shown in Figure 8), where the trailing peak seems to appear less prominently in the latter portion of the Fermi mission than in the earlier data.

To investigate the significance of this effect, we compared the distributions of the observed photon phases before and after MJD 55750 (chosen to maximize the change in the pulse profile's first Fourier coefficient) by calculating the Weighted Kuiper test statistic (Jetsu \& Pelt 1996),

$$
V_{w_{1}, w_{2}}=\max \left\{G_{1}(\Phi)-G_{2}(\Phi)\right\}+\max \left\{G_{2}(\Phi)-G_{1}(\Phi)\right\},
$$

where $G_{1}(\Phi)$ and $G_{2}(\Phi)$ are the empirical weighted cumulative distributions of the photon phases before and after MJD 55750 respectively.

The distribution of $V_{w_{1}, w_{2}}$ under the null hypothesis is unknown, and calculating significances based on the properties of the unweighted statistic always under-estimates the falsealarm rate. To estimate the significance, we therefore performed a Monte Carlo analysis. Using the observed sets of photon weights (before and after MJD 55750), we randomly generated two sets of photon phases, with the jth photon's phase drawn from a common pulse profile ${ }^{14}$ with the

\footnotetext{
14 The distribution of $V_{w_{1}, w_{2}}$ under the null hypothesis should be independent of the chosen pulse profile, since it only tests the possibility that the observed phases are drawn from the same distribution, regardless of the true underlying distribution.
}

probability $p=w_{j}$, otherwise distributed uniformly. This process was repeated many times to estimate the distribution of $V_{w_{1}, w_{2}}$ under the null hypothesis.

From this analysis, we find that the observed value of $V_{w_{1}, w_{2}}$ corresponds to a $p$-value of 0.038 . Given that all 13 pulsars were checked for pulsations, and that a small number of trials were performed when choosing the date defining the boundary between the two intervals, we conclude that this is not a significant variation.

Long-term monitoring of the Fermi-LAT data from this pulsar would be required to detect the presence of any pulse profile mode changes, either by observing another such mode change, or by reducing the uncertainty on the new template pulse profile. A change in pulse profile has only been detected in one gamma-ray pulsar to date, PSR J2021+4026 (Allafort et al. 2013). This variation was accompanied by abrupt changes in the pulsar's gamma-ray flux and spin-down rate, neither of which are observed from PSR J1350-6225.

\section{DISCUSSION}

\subsection{Sensitivity}

Recent publications have argued, both by modeling the emission mechanisms of known radio and gamma-ray pulsars (Perera et al. 2013) and by constructing an unbiased sampling of radio-loud and radio-quiet gamma-ray pulsars (Rubtsov \& Sokolova 2015), that Fermi-LAT should detect significantly more non-recycled gamma-ray pulsars that are radio quiet than are radio loud, by a factor of approximately two. The 13 new pulsars reported here, only 2 of which appear to be radio loud (Paper II) bring the total number of radio-quiet and radio-loud non-recycled gamma-ray pulsars to 51 and 61 respectively. ${ }^{15}$ This would suggest that there are still large numbers of unidentified radio-quiet gamma-ray pulsars requiring blind gamma-ray pulsation searches to be detected. In this section, we compare the newly discovered pulsars to the earlier population of gamma-ray pulsars discovered in blind searches

\footnotetext{
${ }^{15}$ We use the definition from the $2 \mathrm{PC}$ that a radio-loud pulsar has a flux density $S_{1400}>30 \mu \mathrm{Jy}$. Two gamma-ray pulsars have radio detections with lower fluxes, we count them here as radio-quiet.
} 
Table 4

Derived Pulsar Properties

\begin{tabular}{|c|c|c|c|c|c|c|c|}
\hline Pulsar & $l\left(^{\circ}\right)$ & $b\left(^{\circ}\right)$ & $P(\mathrm{~ms})$ & $\begin{array}{l}\dot{P}\left(10^{-15} \mathrm{~s} \mathrm{~s}^{-1}\right) \\
\end{array}$ & $\tau_{\mathrm{c}}(\mathrm{kyr})$ & 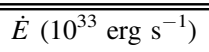 & $\overline{B_{\mathrm{S}}\left(10^{12} \mathrm{G}\right)}$ \\
\hline $\mathrm{J} 0002+6216$ & 117.33 & -0.07 & $115.363568268(2)$ & $5.96703(7)$ & 306 & 153 & 0.8 \\
\hline $\mathrm{J} 0359+5414$ & 148.23 & +0.88 & $79.427232292(1)$ & $16.73359(7)$ & 75 & 1318 & 1.2 \\
\hline $\mathrm{J} 0631+0646$ & 204.68 & -1.24 & $110.9789432160(7)$ & $3.61723(2)$ & 486 & 104 & 0.6 \\
\hline $\mathrm{J} 1057-5851$ & 288.61 & +0.80 & $620.3650313(1)$ & $100.583(5)$ & 98 & 17 & 8.0 \\
\hline $\mathrm{J} 1105-6037$ & 290.24 & -0.40 & 194.938267113(3) & $21.83720(6)$ & 141 & 116 & 2.1 \\
\hline $\mathrm{J} 1350-6225$ & 309.73 & -0.34 & $138.157778213(1)$ & $8.88352(4)$ & 246 & 133 & 1.1 \\
\hline $\mathrm{J} 1528-5838$ & 322.17 & -1.75 & $355.686622097(8)$ & $24.7586(2)$ & 228 & 22 & 3.0 \\
\hline $\mathrm{J} 1623-5005$ & 333.72 & -0.31 & $85.0721461635(8)$ & $4.16118(2)$ & 324 & 267 & 0.6 \\
\hline $\mathrm{J} 1624-4041$ & 340.56 & +6.15 & $167.861145148(1)$ & $4.72489(2)$ & 563 & 39 & 0.9 \\
\hline $\mathrm{J} 1650-4601$ & 339.78 & -0.95 & $127.122893310(2)$ & $15.14468(6)$ & 133 & 291 & 1.4 \\
\hline $\mathrm{J} 1827-1446$ & 17.08 & -1.50 & $499.18661037(3)$ & $45.3351(9)$ & 174 & 14 & 4.8 \\
\hline $\mathrm{J} 1844-0346$ & 28.79 & -0.19 & $112.85464991(1)$ & $154.7031(6)$ & 12 & 4249 & 4.2 \\
\hline $\mathrm{J} 2017+3625$ & 74.51 & +0.39 & $166.7491790419(8)$ & $1.35985(2)$ & 1943 & 12 & 0.5 \\
\hline
\end{tabular}

Note. Columns 2 and 3 give the pulsars' Galactic longitudes $(l)$ and latitudes $(b)$ respectively. Columns 4 and 5 give the derived spin period $(P=1 / f)$ and period derivative $\left(\dot{P}=-\dot{f} / f^{2}\right)$. Characteristic ages, $\tau_{\mathrm{c}}$, spin-down luminosities, $\dot{E}$, and surface magnetic field strengths, $B_{\mathrm{S}}$, are calculated as described in Abdo et al. (2013).

to identify and discuss the overall trends in blind search sensitivity.

One question that we may like to address is, how bright does an unidentified gamma-ray pulsar need to be in order to be detectable in a blind search? In particular, we would like to know the lowest point-source significance within which pulsations can be detected, and how this threshold changes as more data are accumulated.

In Figure 9, we have plotted the point-source significance versus the $H$-test value for each pulsar in the $2 \mathrm{PC}$, as well as the blind search pulsars detected after this catalog was produced (Pletsch et al. 2013), and those discovered by this survey (including PSR J1906+0722, Clark et al. 2015). We can see that the $H$-test value for a pulsar can be well approximated by its point-source TS value (as shown by the dashed line in Figure 9). The values plotted here for pulsars detected in previous blind searches have been scaled back to represent their value at the time of their discovery.

We begin by looking at how the coherent detection statistic, $\mathcal{P}_{1}$, varies with the observation duration, $T_{\text {obs }}$. Since the $H$-test is a maximized sum over $\mathcal{P}_{n}$ values, the relevant scalings with respect to the observation time will be unchanged. As shown in Pletsch \& Clark (2014), the expected values of $\mathcal{P}_{1}$ for a signal with average photon arrival rate, $\mu$, pulse profile Fourier coefficients, $\left\{\gamma_{n}\right\}$, and pulsed fraction $p_{\mathrm{s}}$ over an observation lasting $T_{\mathrm{obs}}$ is approximately

$$
E_{p}\left[\mathcal{P}_{1}\right] \approx 2 p_{\mathrm{s}}^{2} \mu\left|\gamma_{1}\right|^{2} T_{\mathrm{obs}}+2
$$

This shows the well-known result that $\mathcal{P}_{1}$ (and hence $H$ ) increases linearly with time. This is relevant for detecting gamma-ray pulsations using known radio or X-ray ephemerides; since only a small number of trials are required, fully coherent searches are perfectly feasible and signals only need to overcome a low threshold to be detected. As $T_{\text {obs }}$ increases, so too does the point-source significance, and pulsars whose gamma-ray pulsations are not initially above the detection threshold will eventually be detectable. Indeed, pulsations have been detected in this way from sources all the way down to the point-source detection threshold (Hou et al. 2014).

However, the limiting factor in our blind searches is the sensitivity of the initial semicoherent stage. The expected semicoherent (power) $\mathrm{S} / \mathrm{N}$, given a lag-window length, $T$, is

$$
\theta_{S_{1}}^{2}=E_{p}\left[S_{1}\right] \approx p_{\mathrm{s}}^{2} \mu\left|\gamma_{1}\right|^{2} T^{1 / 2} T_{\mathrm{obs}}^{1 / 2} .
$$

The semicoherent $\mathrm{S} / \mathrm{N}$ accumulates much more slowly, only with the square root of $T_{\mathrm{obs}}$. Substituting this into Equation (22), we can identify the effective coherent threshold, $\mathcal{P}_{1}^{*}$, in terms of the semicoherent $\mathrm{S} / \mathrm{N}$ threshold, $S_{1}^{*}$, as

$$
\mathcal{P}_{1}^{*} \approx 2 S_{1}^{*}\left(\frac{T_{\mathrm{obs}}}{T}\right)^{\frac{1}{2}}+2 .
$$

This reveals an unintuitive result: with a fixed semicoherent threshold and lag-window size, as the observation time increases our sensitivity threshold in terms of the coherent signal power (and hence point-source significance) actually increases.

Equation (23) also reveals the solution to this problem: if we are to maintain the same source significance threshold in searches using longer observation times, we must also increase the lag-window duration by the same factor. However, as was derived in Pletsch \& Clark (2014), the computational cost associated with a blind semicoherent search scales with $T^{4} T_{\text {obs }}$.

In Figure 10, we have estimated the semicoherent $\mathrm{S} / \mathrm{N}$ at the time of discovery for all gamma-ray pulsars detected in previous blind searches by calculating their $\mathcal{P}_{1}$ values from the data provided by the $2 \mathrm{PC}$, and scaling these down to the $T_{\mathrm{obs}}$ used in each search. We have also estimated the computational cost that would be required to perform each search (only covering the young pulsar parameter space) using the original lag-window size $\left(T=2^{20} \mathrm{~s}\right)$ and observation length, but with otherwise the same search scheme described in Section 2. Searches prior to Pletsch et al. (2012a) only searched for pulsations from the LAT point-source location, rather than searching over many possible sky locations. While this significantly decreases the required computational cost, this requires pulsars either to be close to the LAT source's estimated position, or to have a strong pulsed signal, such that they can be detected despite a large positional offset. This also rules out the detection of isolated MSPs, whose high frequency requires us to search sky locations with much finer resolution. We therefore exclude the cost of searching for MSPs from our estimated computing costs. 

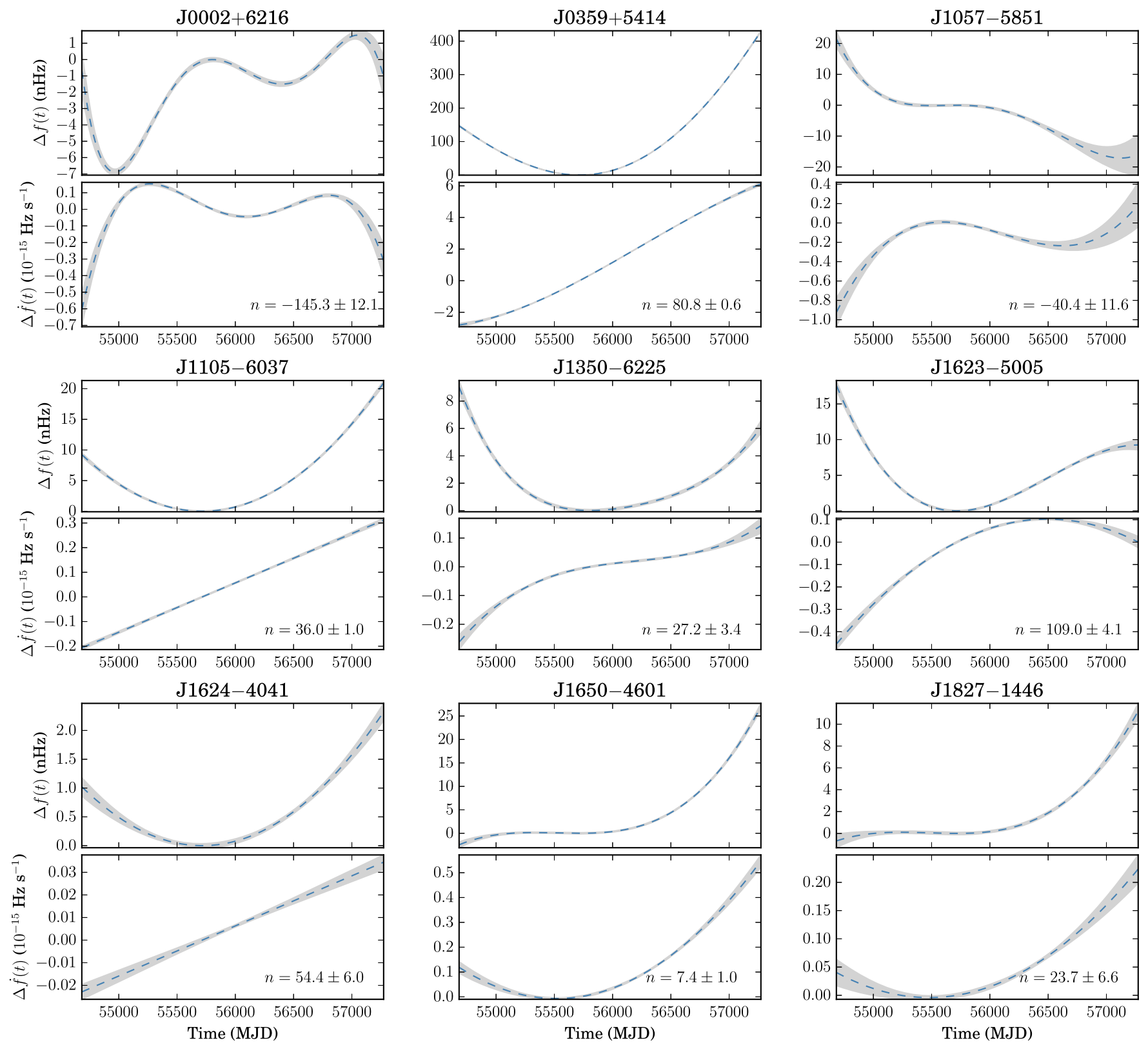

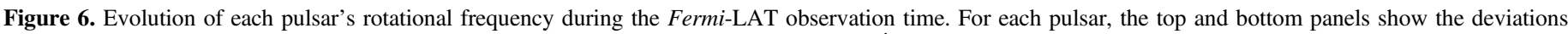

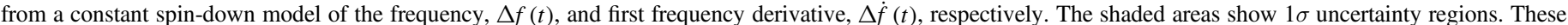

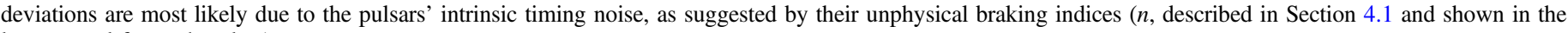
lower panel for each pulsar).

In this figure, we have also estimated the computational cost and $\mathrm{S} / \mathrm{N}$ that each of the new pulsars would have had if we had performed these searches with the lag-window duration used by previous searches. At least four, and possibly as many as seven, pulsars would have been below the lowest semicoherent $\mathrm{S} / \mathrm{N}$ from a previously detected pulsar, and therefore most likely would not have been detected had we not performed the more expensive search with the longer lag window.

Figure 10 also shows how the computing cost and $\mathrm{S} / \mathrm{N}$ increased for these pulsars as a result of using a lag-window with double the length. We see that only one pulsar detected here required less computing time than any previously detected gamma-ray pulsar, with the most expensive detection being more than 100 times more costly. Based on this argument, the current survey should have taken many years to complete, even taking into account a generous estimate of a doubling of the computing power between the two surveys computed on Einstein@Home. In actuality, the first 118 sources were searched in just over one year, similar in duration to the first gamma-ray pulsar survey. We consider this a testament to the improved efficiency of the search methods developed in Pletsch \& Clark (2014).

The signal detected here with the lowest coherent power was from PSR J1105-6037 with $\mathcal{P}_{1}=165$. From the spread about the diagonal of Figure 9, we estimate that a pulsar with a particularly narrow pulse profile should be detectable with a TS of around 100 (a significance of approximately $10 \sigma$ ). The lowest-significance point source in which a pulsar was detected 

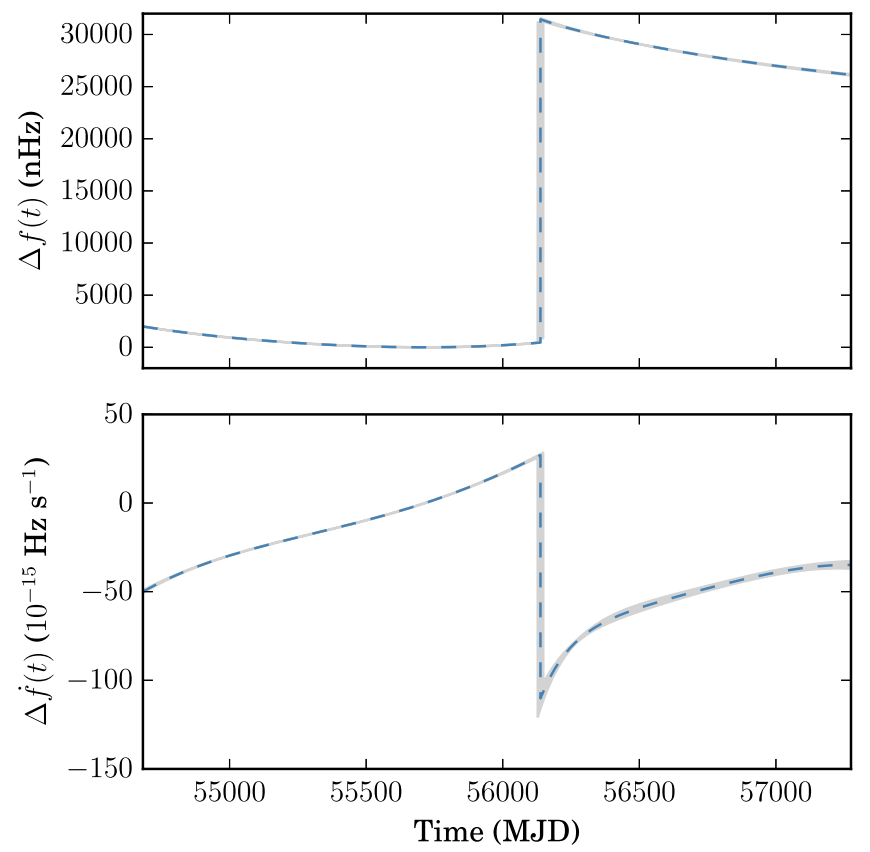

Figure 7. Evolution of the rotational frequency of PSR J1844-0346 during the Fermi-LAT observation time, as in Figure 6.

Table 5

PSR J1844-0346 Glitch Parameters

\begin{tabular}{lc}
\hline \hline Parameter & Value \\
\hline Glitch epoch, $t_{\mathrm{g}}(\mathrm{MJD})$ & $56135(7)$ \\
Frequency increment, $\Delta f_{\mathrm{g}}(\mathrm{Hz})$ & $3.06(1) \times 10^{-5}$ \\
$\dot{f}$ increment, $\Delta \dot{f}_{\mathrm{g}}\left(\mathrm{Hz} \mathrm{s}^{-1}\right)$ & $-9.4(3) \times 10^{-14}$ \\
$\ddot{f}$ increment, $\Delta \ddot{f}_{\mathrm{g}}\left(\mathrm{Hz} \mathrm{s}^{-2}\right)$ & $-7.0(9) \times 10^{-22}$ \\
Decaying $f$ increment, $\Delta f_{D, \mathrm{~g}}(\mathrm{~Hz})$ & $4.5(7) \times 10^{-7}$ \\
Decay time constant, $\tau_{D, \mathrm{~g}}(\mathrm{days})$ & $117(22)$ \\
\hline
\end{tabular}

Note. Reported values of glitch parameters are the mean values obtained from the Monte-Carlo analysis described in Section 4 with $1 \sigma$ uncertainties in the final digits given in brackets.

here (PSR J1350-6225) had a TS of 356.6 (approximate significance $19 \sigma$ ). This could be because the ranking method for our target sources depends on the sources' significance; brighter Fermi-LAT sources were searched earlier than dimmer sources with similar spectral properties. It is therefore possible that, as our survey continues, we may yet detect a pulsar with a lower point-source significance.

In any case, the following trend is clear: as the Fermi mission continues, dimmer pulsars will gradually become detectable to blind searches, but the computing cost required to detect equally low $H$-test signals will rise quickly as we search longer data sets. This survey received a large boost to its sensitivity in the form of the "free" (in terms of computational cost) increase in the observed photon counts offered by the Pass 8 data reprocessing (Atwood et al. 2013). It is unlikely that such a welcome jump in sensitivity will occur again in the near future, and therefore it may become necessary to make some sacrifices to maintain the current search sensitivity without the computing cost requirements becoming unrealistic. For example, reducing the spin-down range to which we are sensitive by a factor of $\sim 10$ will reduce the computational cost by the same factor, while only losing sensitivity to the most

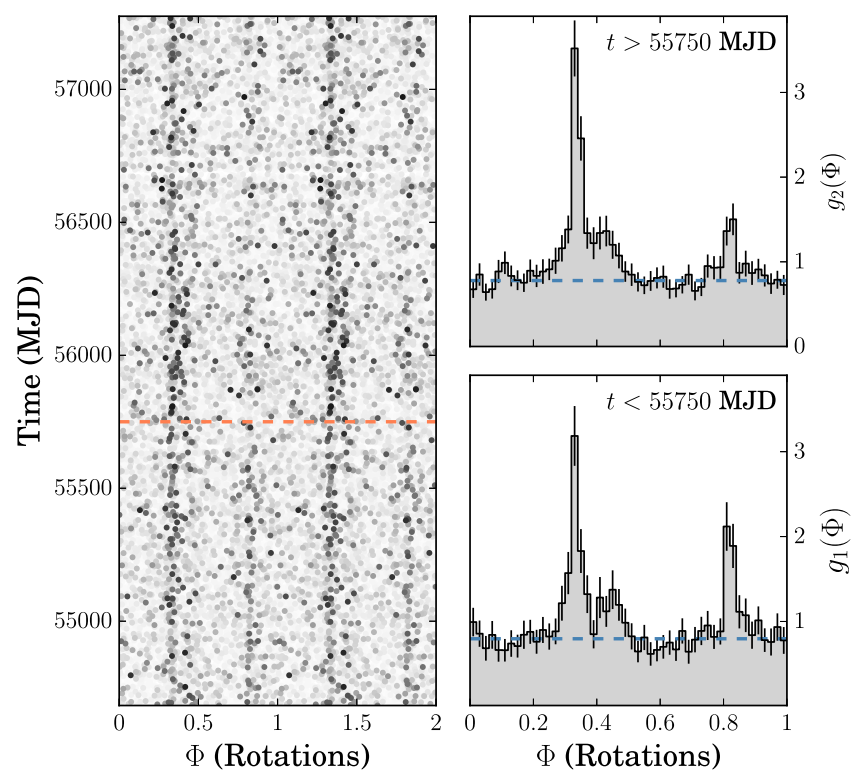

Figure 8. Pulse profile of PSR J1350-6225 showing a possible change around MJD 55750. Left panel: phase-time diagram of the detected photons from PSR J1350-6225, with photon probability weight indicated by the intensity. MJD 55750 is marked by the dashed orange line. Right panels, bottom and top: normalized phase histograms showing the total pulse profile before $\left(g_{1}(\Phi)\right)$ and after MJD $55750\left(g_{2}(\Phi)\right)$ respectively. The estimated background levels, $b$, are shown by the dashed blue line.

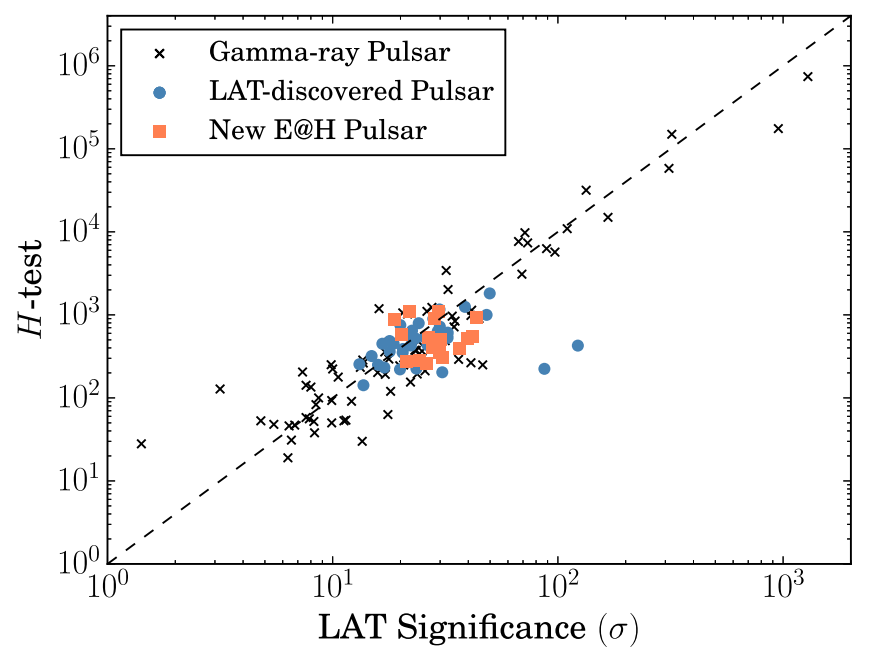

Figure 9. Point-source significance vs. $H$-test value for all pulsars in $2 \mathrm{PC}$, and those detected by the Einstein @ Home survey. The dashed line denotes where $H=$ TS. The $H$-test values and significances for previously detected blind search pulsars have been scaled back from their $2 \mathrm{PC}$ values to estimate their value in the data used in their original searches.

energetic of pulsars. Indeed, one could argue that we already have greatly reduced sensitivity to these pulsars since they typically exhibit the most timing noise and large glitches.

Another option would be to focus our available computing resources to perform more sensitive searches of only the most promising target sources, rather than performing wide-ranging surveys of many unidentified sources as was done here. Indeed, if we look at how the computing cost was distributed among sources, as shown in Figure 4, we can see that, even with a favorable pulse profile, some sources would require a pulsed fraction far higher than unity for us to detect pulsations. The procedures described in Section 3 allow us to reliably predict our search sensitivity to new sources for the first time. Now that 


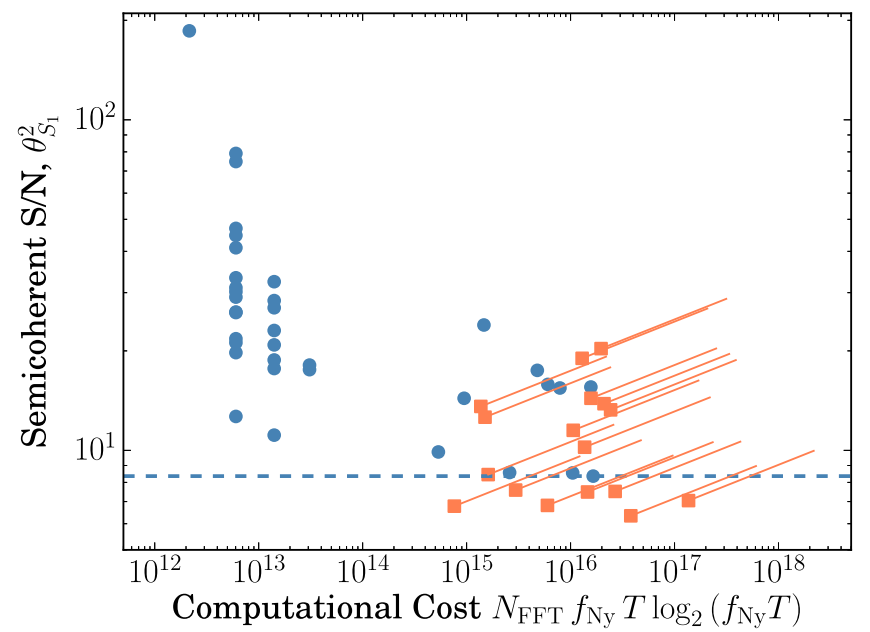

Figure 10. Computing cost vs. semicoherent signal-to-noise ratio comparison for all LAT-discovered gamma-ray pulsars. Those newly discovered in this work are shown by orange squares, previously detected pulsars are shown as blue circles. The locations on this plot of the newly detected pulsars represent their expected location if our survey had used the $50 \%$ shorter lag-window used in previous searches. The lowest semicoherent $\mathrm{S} / \mathrm{N}$ for all blind search pulsars is given by the blue dashed line, we consider signals below this line to be undetectable. The orange diagonal lines show how the computational cost and $\mathrm{S} / \mathrm{N}$ increased for the newly discovered pulsars due to the longer lag window used in this survey.

we are able to predict this in advance, we can remove such sources from our search list and focus our efforts on improving our sensitivity to the more promising sources. If we relax the requirement of $95 \%$ detection probability used in producing Figure 4 slightly to reflect a more moderate chance of detecting pulsations from each source, we find that $\sim 25 \%$ of the computing cost of this survey could perhaps have been better spent by removing unpromising sources.

\subsection{New Pulsars}

Only energetic pulsars with spin-down powers above $10^{33} \mathrm{erg} \mathrm{s}^{-1}$ are observed to emit gamma-ray pulsations (Guillemot et al. 2016). The group of non-recycled gamma-ray pulsars, therefore, lies at the young and energetic end of the overall pulsar population, with typical characteristic ages of less than a few million years, and surface magnetic field strengths between $10^{11}-10^{13} \mathrm{G}$. This contrasts with the population of nonrecycled radio pulsars (which extends to characteristic ages of up to $1 \mathrm{Gyr}$, and spin-down powers as low as $10^{30} \mathrm{erg} \mathrm{s}^{-1}$, Manchester et al. 2005) and with magnetars (whose surface magnetic fields are in excess of $10^{14} \mathrm{G}$, Turolla et al. 2015). In these respects, the group of pulsars detected here appears to be consistent with the overall population of young gamma-ray pulsars (see Figure 1, and Table 4).

Two of the new pulsars reported here (PSR J1057-5851 and PSR J1827-1446) are the most slowly spinning gammaray pulsars yet discovered, at $1.6 \mathrm{~Hz}$ and $2.0 \mathrm{~Hz}$ respectively. While the existence of these pulsars is not in tension with estimates of the gamma-ray pulsar "death line" (Wang \& Hirotani 2011), their discovery does extend the known population of gamma-ray pulsars down to lower spin frequencies. Sensitive blind searches that can detect faint young pulsars are important to fully explore the low- $\dot{E}$ region of the gamma-ray pulsar population, and to reduce biases inherent in it (Guillemot et al. 2016).
Also of interest are the pulsars detected here despite exhibiting significant timing noise, PSR J1844-0346, and PSR J0359+5414. The large contributions of higher frequency derivatives mean that the original phase model used in the blind search could not maintain phase coherence over the full duration of the data. These effects make it very difficult for the coherent follow-up stages to pick up these signals. On the other hand, the semicoherent stage is largely unaffected by timing noise; though, large glitches are also detrimental to the semicoherent sensitivity. Noisy pulsars will therefore only appear as semicoherent candidates, and may easily escape detection from our pipeline, which focuses on the results from the final coherent follow-up. Further investigation of the vast number of semicoherent candidates reported by Einstein@ Home may yet reveal more noisy pulsars lurking in our results.

\section{CONCLUSIONS}

We have presented the discovery of 13 new gamma-ray pulsars found by the ongoing Einstein@Home survey of unidentified Fermi-LAT sources. Among these pulsars are two new energetic pulsars with $\dot{E}>10^{36} \mathrm{erg} \mathrm{s}^{-1}$, one of which experienced a large glitch; and the two slowest spinning gamma-ray pulsars yet detected.

As the Fermi mission continues and the LAT gathers more data, the sensitivity to weak pulsar signals will increase, and many currently undetectable pulsars could rise above the detection threshold in the near future; though, future searches with more data will also require even more computing power to be sensitive to similarly weak signals.

We also placed realistic upper limits on the pulsed flux from point sources from which no pulsations were detected. The framework for this allows us to also predict our sensitivity to other sources, enabling us to identify promising targets for searching, and also to veto sources from which pulsations would be almost impossible to detect. This will allow us to focus our computing power on increasing our sensitivity to the most promising sources in future surveys.

A further exciting new advancement is the recent launch of the first Einstein@Home survey for gamma-ray pulsars in candidate binary systems with well-constrained orbital parameters, similar to the search that discovered PSR J1311-3430 (Pletsch et al. 2012). The additional computing power of Einstein@Home will enable more complicated searches, allowing for searches from sources with larger uncertainties in their orbital parameters, or even with slight eccentricities.

We are extremely grateful to all volunteers who have donated their CPU time to the Einstein@Home project, without whom this survey could not have been performed. We are especially grateful to those users whose computers discovered the 13 new pulsars reported here. They are as follows. ${ }^{16}$

1. PSR J0002+6216: James Drews of UW-Madison, WI, USA and Ralph Elwell of Richland, WA, USA;

2. PSR J0359+5414: Whelton A. Miller III, Lincoln University of Pennsylvania \& University of Pennsylvania, USA; the ATLAS Cluster, AEI, Hannover, Germany and Philip "Delty" Horney of the GPU Users Group, Fort Wright, KY, USA;

\footnotetext{
16 Where the volunteer's name is unknown or private, we give the Einstein@Home username in quotation marks.
} 
3. PSR J0631+0646: Katagiri, Atsushi of Kawasaki, Japan and Nicholas Huwar of Houston, TX, USA;

4. PSR J1057-5851: Syracuse University HTC Campus Grid, ${ }^{17}$ NY, USA; Igor Yakushin of Chicago, IL, USA and the LIGO Laboratory, USA

5. PSR J1105-6037: The ATLAS Cluster, AEI, Hannover, Germany and Syracuse University HTC Campus Grid (see footnote 16), NY, USA;

6. PSR J1350-6225: Petr Ruzicka of Brno, Czech Republic and Bryden Kanngiesser of Calgary, Canada;

7. PSR J1528-5838: "fred c" and Gabriel Vasquez of Miami, FL, USA;

8. PSR J1623-5005: Lars Bollwinkel, of Kiel, Germany and Greg Dorais of Martinez, CA, USA;

9. PSR J1624-4041: Xio of NYC and Hung Tran of Chandler, AZ, USA;

10. PSR J1650-4601: Syracuse University HTC Campus Grid (see footnote 16), NY, USA and Eric Schwartz of Vashon Island, WA, USA;

11. PSR J1827-1446: The ATLAS Cluster, AEI, Hannover, Germany; Igor Yakushin of Chicago, IL, USA and the LIGO Laboratory, USA

12. PSR J1844-0346: Aurélien FAUCHEUX of Antibes, France and Roger Gulbranson, Ph.D. of Wickliffe, $\mathrm{OH}, \mathrm{USA}$;

13. PSR J2017+3625: Kurt Gramoll, Ph.D., University of Oklahoma, OK, USA and Michael Brandau, of Kassel, Germany.

This work was supported by the Max-Planck-Gesellschaft (MPG), by the Deutsche Forschungsgemeinschaft (DFG) through an Emmy Noether research grant PL 710/1-1 (PI: Holger J. Pletsch), and by NSF award 1104902.

The Fermi-LAT Collaboration acknowledges generous ongoing support from a number of agencies and institutes that have supported both the development and the operation of the LAT as well as scientific data analysis. These include the National Aeronautics and Space Administration and the Department of Energy in the United States, the Commissariat à l'Energie Atomique and the Centre National de la Recherche Scientifique/Institut National de Physique Nucléaire et de Physique des Particules in France, the Agenzia Spaziale Italiana and the Istituto Nazionale di Fisica Nucleare in Italy, the Ministry of Education, Culture, Sports, Science and Technology (MEXT), High Energy Accelerator Research Organization (KEK) and Japan Aerospace Exploration Agency (JAXA) in Japan, and the K. A. Wallenberg Foundation, the Swedish Research Council and the Swedish National Space Board in Sweden.

Additional support for science analysis during the operations phase is gratefully acknowledged from the Istituto Nazionale di Astrofisica in Italy and the Centre National d'Études Spatiales in France.

\section{APPENDIX A CANDIDATE RANKING}

In the final follow-up stages, performed offline, we would like to only search the most significant candidates. To rank candidates by their significance, we need to account for the effective number of trials from which each candidate has

\footnotetext{
${ }^{17}$ Supported by NSF awards ACI-1341006 and ACI-1541396.
}

resulted. While the number of (semicoherent) independent trials is approximately the same in each work unit within each source, there are more work units in higher frequency bands due to the density of sky locations increasing with frequency. Additionally, since the grid of sky locations searched in the first stage is constructed at the highest frequency in the band, whereas the "zoomed in" grids are defined by the candidates' spin frequencies, the number of trials in the refinement step varies from candidate to candidate.

The overall result of these effects is that candidates with high detection statistic values are more likely to occur by chance in higher frequency bands than in lower frequency bands, and at the higher end of the frequency band. We construct a consistent ranking statistic by comparing candidates' false-alarm probabilities while taking the differing number of trials into account.

We start from the result (Kruger et al. 2002) that the cumulative distribution function (cdf), $G\left(X_{\max }\right)$, of the maximum value of $N$ samples of the random variable $X$, is related to $F(X)$, the cdf of $X$, by

$$
G\left(X_{\max }\right)=\left[F\left(X_{\max }\right)\right]^{N} .
$$

The false-alarm probability of $X_{\max }$ after $N$ samples is therefore

$$
\begin{aligned}
P_{\mathrm{FA}, \mathrm{N}}\left(X_{\mathrm{max}}\right) & =1-\left[1-P_{\mathrm{FA}, 1}\left(X_{\max }\right)\right]^{N} \\
& \approx N P_{\mathrm{FA}, 1}\left(X_{\max }\right),
\end{aligned}
$$

where we have assumed that $P_{\mathrm{FA}, 1} \ll 1 / N$. In our case, the single-trial false alarm for a candidate with coherent power $\mathcal{P}_{1}$ is

$$
\begin{aligned}
P_{\mathrm{FA}, 1}\left(\mathcal{P}_{1}\right) & =\int_{\mathcal{P}_{1}}^{\infty} \chi_{2}^{2}\left(\mathcal{P}_{1}^{\prime}\right) d \mathcal{P}_{1}^{\prime} \\
& =e^{-\mathcal{P}_{1} / 2},
\end{aligned}
$$

where $\chi_{2}^{2}(X)$ is the central chi-squared distribution with two degrees of freedom.

It is considerably more difficult to estimate the effective number of independent trials, since each candidate is the result of a large number of trials in previous search stages using different detection statistics. However, since at this stage we are only interested in ranking candidates within each source, and the number of independent trials in the semicoherent step is approximately the same for each candidate, we only need to consider the varying number of trials in the follow-up stages, and the total number of work units in each frequency band.

The overall false-alarm probability is therefore a function of the frequency of the candidate, $f$, and the coherent power:

$$
P_{\mathrm{FA}}\left(\mathcal{P}_{1}, f\right)=K N_{\mathrm{W}}(f) N_{\mathrm{F}}(f) e^{-\mathcal{P}_{1} / 2},
$$

where the constant of proportionality, $K$, is the (unknown) number of independent trials per work unit, $N_{W}(f)$ is the number of work units within the appropriate frequency band and $N_{F}(f)$ is the number of trials in the coherent follow-up stage for a candidate at frequency $f$.

We define the ranking statistic, $\hat{R}$ for follow-up analyses according to the logarithm of the inverse of the false-alarm probability,

$$
\begin{aligned}
\hat{R}\left(\mathcal{P}_{1}, f\right) & \equiv-\log \left[\frac{P_{\mathrm{FA}}\left(\mathcal{P}_{1}, f\right)}{K}\right] \\
& =\frac{\mathcal{P}_{1}}{2}-\log \left[N_{W}(f)\right]-\log \left[N_{F}(f)\right],
\end{aligned}
$$



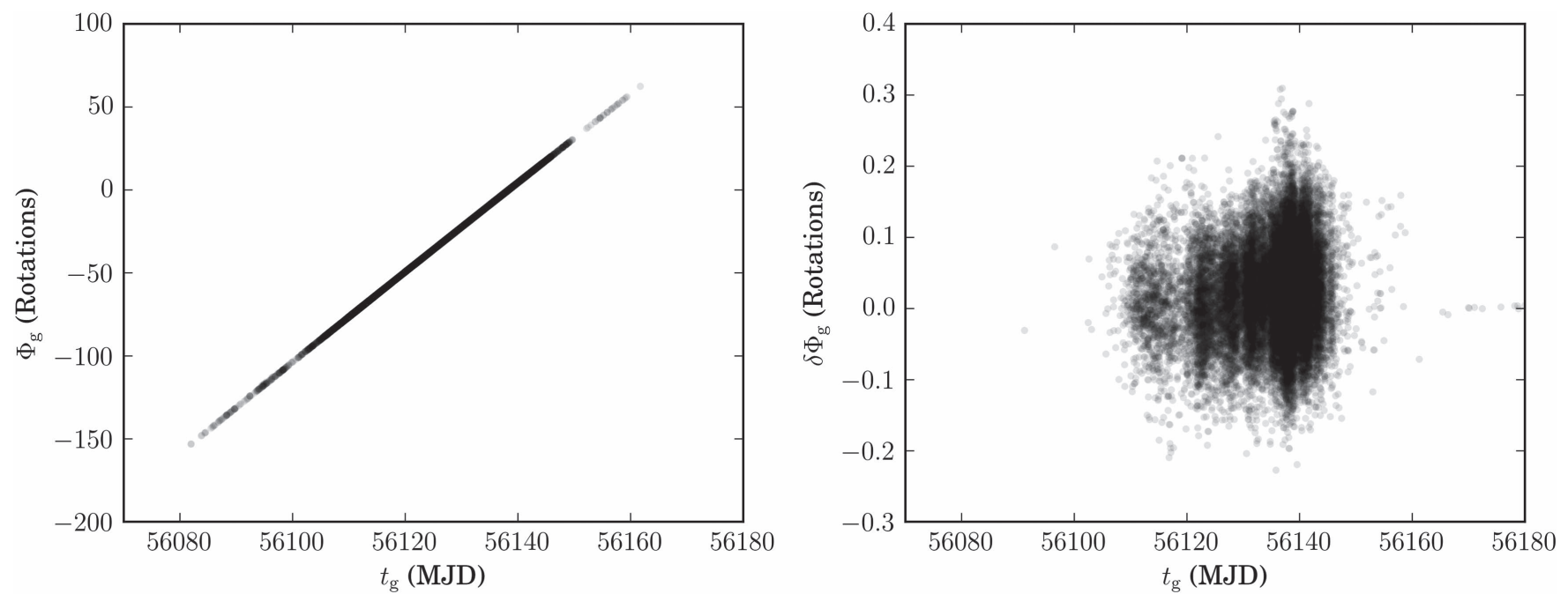

Figure 11. Glitch epochs and phase increments from Monte Carlo sampling during timing of PSR J1844-0346. In the left panel, the phase increment was completely free to vary and exhibits a high correlation with the glitch epoch. In the right panel, the glitch epoch-dependent part of the phase increment was accounted for, and a small random increment was allowed to vary on top of this, removing the correlation.

where we have removed the constant term corresponding to $K$. We note that the above formulation of $\hat{R}$ can only be used to rank pulsar candidates from the same source, as the effective number of independent trials per work unit $(K)$ varies from source to source.

\section{APPENDIX B}

\section{DISTRIBUTION OF $S_{1}$ WITH A SIGNAL}

To estimate the sensitivity of the search, it is necessary to know the expected distribution of $\hat{S_{1}}$ in the presence of a signal for each source. To derive this, we first expand the double sum of Equation (2) and separate it into terms in which photon indices are never equal. Identifying the photon weights as the probability that each photon originated from the source in question, and therefore assuming that each photon has a probability $w_{j} p_{\mathrm{s}}$ of having been pulsed, we find the following expressions,

$$
\begin{gathered}
E_{p}\left[w_{j}^{m} e^{-i n \Phi\left(t_{j}\right)}\right]=w_{j}^{m+1} p_{\mathrm{s}} \gamma_{n}, \\
E_{p}\left[w_{j}^{m} e^{i n \Phi\left(t_{j}\right)}\right]=w_{j}^{m+1} p_{\mathrm{s}} \gamma_{n}^{*} .
\end{gathered}
$$

The expectation value and variance of $S_{1}$ in the presence of a signal are therefore found, after some relabeling and rearranging, to be

$$
\begin{aligned}
E_{p}\left(p_{\mathrm{s}},\left\{\gamma_{n}\right)\left[S_{1}\right]=\right. & \frac{p_{\mathrm{s}}^{2}\left|\gamma_{1}\right|^{2}}{\kappa_{S_{1}}} \sum_{j=1}^{N} \sum_{k \neq j}^{N} w_{j}^{2} w_{k}^{2} \hat{W}_{T}^{\text {rect }}\left(\tau_{j k}\right), \\
\sigma_{p}^{2}\left(p_{\mathrm{s}},\left\{\gamma_{n}\right)\left[S_{1}\right]=\right. & \frac{1}{\kappa_{S_{1}}^{2}} \sum_{j=1}^{N} \sum_{k \neq j}^{N}\left[w_{j}^{2} w_{k}^{2} \hat{W}_{T}^{\text {rect }}\left(\tau_{j k}\right)\right. \\
& \times\left(1+p_{\mathrm{s}}^{2}\left|\gamma_{2}\right|^{2} w_{j} w_{k}\right. \\
& -2 p_{\mathrm{s}}^{4}\left|\gamma_{1}\right|^{4} w_{j}^{2} w_{k}^{2} \\
& +\sum_{l \neq j \neq k}^{N} w_{l}^{2} \hat{W}_{T}^{\text {rect }}\left(\tau_{j l}\right)\left[2 p_{\mathrm{s}}^{2}\left|\gamma_{1}\right|^{2}\right. \\
& +2 p_{\mathrm{s}}^{3} \mathfrak{R}\left(\gamma_{2}\left(\gamma_{1}^{*}\right)^{2}\right) w_{j} \\
& \left.\left.\left.-4 p_{\mathrm{s}}^{4}\left|\gamma_{1}\right|^{4} w_{j}^{2}\right]\right)\right] .
\end{aligned}
$$

The expected semicoherent $\mathrm{S} / \mathrm{N}$ for a signal with pulsed fraction $p_{\mathrm{s}}$ and a pulse profile with Fourier coefficients $\left\{\gamma_{n}\right\}$ is therefore

$$
\begin{aligned}
\theta_{S_{1}}^{2}\left(p_{\mathrm{s}},\left\{\gamma_{n}\right)\right. & =\frac{E_{p}\left[S_{1}\right]-E_{0}\left[S_{1}\right]}{\sqrt{\sigma_{0}^{2}\left[S_{1}\right]}} \\
& =p_{\mathrm{s}}^{2}\left|\gamma_{1}\right|^{2} \sqrt{\sum_{j=1}^{N} \sum_{k \neq j}^{N} w_{j}^{2} w_{k}^{2} \hat{W}_{T}^{\text {rect }}\left(\tau_{j k}\right) .}
\end{aligned}
$$

In addition to the statistical variance of $S_{1}$, a signal at a random location in the parameter space will be detected at the nearest grid point, and some signal power will be lost as a result of this offset. Denoting this mismatch by $m$, the pdf of $\hat{S}_{1}$ is therefore the pdf of the product of $S_{1}$ and $(1-m)$, which we approximate as a Gaussian with the same mean and variance,

$$
\begin{aligned}
E_{p}\left[\hat{S}_{1}\right]= & E_{p}\left[S_{1}(1-m)\right]=E_{p}\left[S_{1}\right](1-E[m), \\
\sigma_{p}^{2}\left[\hat{S_{1}}\right]= & \sigma_{p}^{2}\left[S_{1}\right] \sigma^{2}[m]+\sigma_{p}^{2}\left[S_{1}\right](1-E[m])^{2} \\
& +\sigma^{2}[m] E_{p}\left[S_{1}\right]^{2} .
\end{aligned}
$$

The values of $E[m]$ and $\sigma^{2}[m]$ depend on the geometry of the search grid. For the grid used in this survey, which had a maximum mismatch per parameter of 0.15 and an interbinned frequency spectrum, $E[\mathrm{~m}] \approx 0.22$ and $\sigma^{2}[\mathrm{~m}] \approx 8 \times 10^{-3}$. This pdf is illustrated in Figure 3.

\section{APPENDIX C \\ EFFICIENT SAMPLING OF GLITCH PARAMETERS}

As mentioned in Section 4.2, when timing large glitches, a phase increment occurring at the time of the glitch can be included in the phase model to ensure that the likelihood surface in the glitch epoch is continuous and easy to sample.

However, this increment is strongly correlated with the glitch epoch, as can be seen in the first panel of Figure 11, which can lead to inefficient Monte Carlo sampling. Once an initial combination of valid glitch parameters has been found (including a suitable combination of glitch epoch and phase increment that removes any phase discontinuity at the glitch) 
we can remove this correlation by separating the total phase increment in our phase model into separate terms: a glitch epoch-dependent term accounting for the known difference in phase between the glitch model parameters being sampled and the initial "reference" glitch model, plus a random offset that is allowed to vary as part of the Monte Carlo sampling. Denoting the reference glitch model with the subscript $\mathrm{g}_{0}$, and the sampled glitch model parameters by the subscript $\mathrm{g}_{1}$, the total phase increment is

$$
\Phi_{\mathrm{g}_{1}}=\delta \Phi_{\mathrm{g}_{1}}+\Phi_{\mathrm{g}_{0}}+ \begin{cases}-\Delta \Phi_{\mathrm{g}_{1}}\left(t_{\mathrm{g}_{0}}\right), & t_{\mathrm{g}_{1}}<t_{\mathrm{g}_{0}} \\ \Delta \Phi_{\mathrm{g}_{0}}\left(t_{\mathrm{g}_{1}}\right), & t_{\mathrm{g}_{1}}>t_{\mathrm{g}_{0}}\end{cases}
$$

where, for $i=0,1$,

$$
\begin{aligned}
\Delta \Phi_{\mathrm{g}_{\mathrm{i}}}(t)= & 2 \pi\left[\Delta f_{\mathrm{g}_{\mathrm{i}}}\left(t-t_{\mathrm{g}_{\mathrm{i}}}\right)+\frac{\Delta \dot{f}_{\mathrm{g}_{\mathrm{i}}}}{2}\left(t-t_{\mathrm{g}_{\mathrm{i}}}\right)^{2}\right. \\
& +\frac{\Delta \ddot{\mathrm{g}}_{\mathrm{g}_{\mathrm{i}}}}{6}\left(t-t_{\mathrm{g}_{\mathrm{i}}}\right)^{3} \\
& \left.+\Delta f_{\mathrm{D}, \mathrm{g}_{\mathrm{i}}} \tau_{\mathrm{D}, \mathrm{g}_{\mathrm{i}}}\left(1-e^{\left.-\left(t-t_{\mathrm{g}}\right) / \tau_{\mathrm{D}, \mathrm{g}}\right)}\right)\right]
\end{aligned}
$$

The first term of Equation (34) ensures that the phase increment is free to vary over a small range to find the glitch parameters with the highest likelihood. The other terms ensure that the reference glitch model's desirable property of causing no large phase discontinuity between the pulse before and after the glitch also applies to the sampled glitch parameters. This ensures that the sampling rate (and hence the efficiency of the timing procedure) is not unnecessarily burdened by having to phase-fold the data with glitch models resulting in large phase discontinuities, which will obviously have a low likelihood and be rejected.

Removing the correlation between the glitch epoch and the part of the phase increment that is being sampled ensures that the Monte Carlo chains explore the parameter space efficiently. This is especially helpful at the beginning of the Monte Carlo run, as the starting locations of the chain are spread uniformly throughout the parameter space, and could otherwise easily get stuck in low-likelihood regions, as they struggle to jump to the very narrow, highly correlated region of high likelihood.

\section{REFERENCES}

Abadie, J., Abbott, B. P., Abbott, R., et al. 2010, ApJ, 722, 1504 Abdo, A. A., Ackermann, M., Ajello, M., et al. 2009, Sci, 325, 840 Abdo, A. A., Ackermann, M., Ajello, M., et al. 2010a, ApJL, 710, L92 Abdo, A. A., Ackermann, M., Ajello, M., et al. 2010b, ApJ, 722, 1303 Abdo, A. A., Ackermann, M., Atwood, W. B., et al. 2008, Sci, 322, 1218 Abdo, A. A., Ajello, M., Allafort, A., et al. 2013, ApJS, 208, 17 Acero, F., Ackermann, M., Ajello, M., et al. 2015, ApJS, 218, 23 Acero, F., Ackermann, M., Ajello, M., et al. 2016, ApJS, 223, 26 Ajello, M., Albert, A., Atwood, W. B., et al. 2016, ApJ, 819, 44 Allafort, A., Baldini, L., Ballet, J., et al. 2013, ApJL, 777, L2 Allen, B., Knispel, B., Cordes, J. M., et al. 2013, ApJ, 773, 91
Astone, P., Borkowski, K. M., Jaranowski, P., Pietka, M., \& Królak, A. 2010, PhRvD, 82, 022005

Atwood, W., Albert, A., Baldini, L., et al. in Proc. IV Fermi Symp., eConf C121028, 8 (arXiv:1303.3514)

Atwood, W. B., Abdo, A. A., Ackermann, M., et al. 2009, ApJ, 697, 1071

Atwood, W. B., Ziegler, M., Johnson, R. P., \& Baughman, B. M. 2006, ApJL, 652, L49

Bartels, R., Krishnamurthy, S., \& Weniger, C. 2016, PhRvD, 116, 051102

Camilo, F., Kerr, M., Ray, P. S., et al. 2015, ApJ, 810, 85

Clark, C. J., Pletsch, H. J., Wu, J., et al. 2015, ApJL, 809, L2

Clark, C. J., Pletsch, H. J., Wu, J., et al. 2016, ApJL, 832, L15

Cromartie, H. T., Camilo, F., Kerr, M., et al. 2016, ApJ, 819, 34

de Jager, O. C., Raubenheimer, B. C., \& Swanepoel, J. W. H. 1989, A\&A, 221,180

Dib, R., Kaspi, V. M., \& Gavriil, F. P. 2009, ApJ, 702, 614

Foreman-Mackey, D., Hogg, D. W., Lang, D., \& Goodman, J. 2013, PASP, 125,306

Frigo, M., \& Johnson, S. G. 2005, IEEEP, 93, 216

Goodman, J., \& Weare, J. 2010, Comm. App. Math. Comp. Sci., 5, 65

Guillemot, L., Johnson, T. J., Venter, C., et al. 2012, ApJ, 744, 33

Guillemot, L., Smith, D. A., Laffon, H., et al. 2016, A\&A, 587, A109

H.E.S.S. Collaboration, Abdalla, H., Abramowski, A., et al. 2016, A\&A, in press (arXiv:1609.00600)

Hessels, J. W. T., Ransom, S. M., Stairs, I. H., et al. 2006, Sci, 311, 1901

Ho, W. C. G., \& Heinke, C. O. 2009, Natur, 462, 71

Hobbs, G., Lyne, A. G., \& Kramer, M. 2010, MNRAS, 402, 1027

Hooper, D., Cholis, I., Linden, T., Siegal-Gaskins, J. M., \& Slatyer, T. R. 2013, PhRvD, 88, 083009

Hou, X., Smith, D. A., Guillemot, L., et al. 2014, A\&A, 570, A44

Jetsu, L., \& Pelt, J. 1996, A\&AS, 118, 587

Johnson, T. J., Venter, C., Harding, A. K., et al. 2014, ApJS, 213, 6

Kerr, M. 2010, PhD thesis, Univ. Washington

Kerr, M. 2011, ApJ, 732, 38

Kerr, M., Ray, P. S., Johnston, S., Shannon, R. M., \& Camilo, F. 2015, ApJ, 814,128

Kruger, A. T., Loredo, T. J., \& Wasserman, I. 2002, ApJ, 576, 932

Lopez, L. A., Ramirez-Ruiz, E., Castro, D., \& Pearson, S. 2013, ApJ, 764, 50

Lyne, A. G., Jordan, C. A., Graham-Smith, F., et al. 2015, MNRAS, 446, 857

Lyons, R. G. 2004, Understanding Digital Signal Processing (2nd ed.; Upper Saddle River, NJ: Prentice Hall)

Manchester, R. N., Hobbs, G. B., Teoh, A., \& Hobbs, M. 2005, AJ, 129 1993

Marshall, F. E., Gotthelf, E. V., Zhang, W., Middleditch, J., \& Wang, Q. D. 1998, ApJL, 499, L179

O’Leary, R. M., Kistler, M. D., Kerr, M., \& Dexter, J. 2016, PhRvD, submitted (arXiv:1601.05797)

Perera, B. B. P., McLaughlin, M. A., Cordes, J. M., et al. 2013, ApJ, 776, 61

Pletsch, H. J., \& Allen, B. 2009, PhRvL, 103, 181102

Pletsch, H. J., \& Clark, C. J. 2014, ApJ, 795, 75

Pletsch, H. J., Guillemot, L., Allen, B., et al. 2012a, ApJ, 744, 105

Pletsch, H. J., Guillemot, L., Allen, B., et al. 2012b, ApJL, 755, L20

Pletsch, H. J., Guillemot, L., Allen, B., et al. 2013, ApJL, 779, L11

Pletsch, H. J., Guillemot, L., Fehrmann, H., et al. 2012, Sci, 338, 1314

Ray, P. S., Kerr, M., Parent, D., et al. 2011, ApJS, 194, 17

Rubtsov, G. I., \& Sokolova, E. V. 2015, JETPL, 100, 689

Saz Parkinson, P. M., Dormody, M., Ziegler, M., et al. 2010, ApJ, 725, 571

Schwarz, G. 1978, AnSta, 6, 461

Turolla, R., Zane, S., \& Watts, A. L. 2015, RPPh, 78, 116901

van der Klis, M. 1989, in Timing Neutron Stars, ed. H. Oegelman \& E. Heuvel (Berlin: Springer)

Wang, R.-B., \& Hirotani, K. 2011, ApJ, 736, 127

Welch, P. 1967, IEEE Trans. Audio Electroacoust., 15, 70

Yu, M., Manchester, R. N., Hobbs, G., et al. 2013, MNRAS, 429, 688

Zhu, H., Tian, W. W., \& Zuo, P. 2014, ApJ, 793, 95

Zhu, W., Kaspi, V. M., Dib, R., et al. 2008, ApJ, 686, 520 